\title{
Systematic review of the evidence for Trails B cut-off scores in assessing fitness-to-drive
}

\author{
Mononita Roy, MD, FRCPC, ${ }^{1,2}$ Frank Molnar, MSc, MDCM, FRCPC ${ }^{1-6}$ \\ ${ }^{1}$ Division of Geriatric Medicine, The University of Ottawa, Ottawa, ON; ${ }^{2}$ The Ottawa Hospital, Ottawa, ON; ${ }^{3}$ The Bruyere \\ Research Institute, Ottawa, ON; ${ }^{4}$ The Clinical Epidemiology Program, Ottawa Hospital Research Institute, Ottawa, ON; \\ ${ }^{5}$ The Regional Geriatric Program of Eastern Ontario, The Ottawa Hospital, Ottawa, ON; ${ }^{6}$ CanDRIVE: a CIHR Institute of \\ Aging funded New Emerging Team, Ottawa Hospital Research Institute, Ottawa, ON
}

DOI:http://dx.doi.org/10.5770/cgj.16.76

\section{ABSTRACT}

\section{Background}

Fitness-to-drive guidelines recommend employing the Trail Making B Test (a.k.a. Trails B), but do not provide guidance regarding cut-off scores. There is ongoing debate regarding the optimal cut-off score on the Trails B test.

The objective of this study was to address this controversy by systematically reviewing the evidence for specific Trails B cut-off scores (e.g., cut-offs in both time to completion and number of errors) with respect to fitness-to-drive.

\section{Methods}

Systematic review of all prospective cohort, retrospective cohort, case-control, correlation, and cross-sectional studies reporting the ability of the Trails B to predict driving safety that were published in English-language, peer-reviewed journals.

\section{Results}

Forty-seven articles were reviewed. None of the articles justified sample sizes via formal calculations. Cut-off scores reported based on research include: 90 seconds, 133 seconds, 147 seconds, 180 seconds, and $<3$ errors.

\section{Conclusions}

There is support for the previously published Trails B cut-offs of 3 minutes or 3 errors (the ' 3 or 3 rule'). Major methodological limitations of this body of research were uncovered including (1) lack of justification of sample size leaving studies open to Type II error (i.e., false negative findings), and (2) excessive focus on associations rather than clinically useful cut-off scores.
Key words: Trail Making Test, Trails B, driving, fitness-todrive, cut-off

\section{INTRODUCTION}

Physicians in most Canadian jurisdictions are legally mandated to report medical findings that could impact on fitnessto-drive (http://www.cma.ca/driversguide). ${ }^{(1)}$ Even where reporting is not mandatory, physicians can still potentially be found liable if they fail to report a patient who harms others due to a car crash attributed to their medical impairments. ${ }^{(2)}$ On a more positive note, the reporting of medical findings that could impact on fitness-to-drive also represents an opportunity to fulfill an important societal role; assessments of fitness-to-drive allow physicians to help their patients avoid disabling injury or death and also to help patients and their families avoid the grief and legal repercussions associated with contributing to the injuries or deaths of other road users or bystanders. ${ }^{(2)}$

Driving guidelines such as those of the Canadian Medical Association, the Canadian Council of Motor Transport Administrators, the Driver and Vehicle Licensing Agency in the United Kingdom, and the American Medical Association recommend the Trail Making B Test (a.k.a. Trails B) to assess fitness-to-drive. ${ }^{(1,3,4,5)}$ Trails B tests dual attention (cognitive flexibility in switching attention between two competing static sets of stimuli which is a much lower level of cognitive demand than switching between multiple moving stimuli encountered when driving) and executive function. Driving represents a "super-Instrumental Activity of Daily Living (super-IADL)" or "super-executive function" that can result in death if performed incorrectly or too slowly - this, along with the risk to others, makes it unique among IADLs or executive functions. Unfortunately, guidelines rarely advise physicians regarding which Trails B findings indicate unfitness-to-drive.

A study by Tombaugh ${ }^{(6)}$ of the normative values of the Trails B test demonstrated that the mean time to complete Trails B is $<180$ seconds for all age groups. There were 
some outliers whose scores exceeded 180 seconds; the lowest 20th percentile in the 80 to 84 age group and the lowest 30th percentile in the 85 to 89 age group, but the validity of the latter findings is questionable given the small sample size in these age-specific cells. It is also possible that some of these findings do not represent true normative values (i.e., values for persons without diseases or drugs affecting the results), but may represent hidden disease or hidden medication effects. (7) Even if these are true norms for healthy people, being in a normative range may not necessarily mean the patient is safe to drive. We have to accept reality-as people get older, they do not have more time to stop their cars or to respond to emergencies. Physical laws do not change according to age. We must, therefore, remain very skeptical of age-adjusted norms for tests used to screen for fitness-to-drive. ${ }^{(7)}$

Continuing medical education articles have recommended a Trails B cut-off of 180 seconds or three errors (i.e., 3 minutes or 3 errors; the ' 3 or 3 rule'). ${ }^{(2,7,8)}$ Given the findings of Tombaugh, ${ }^{(6)}$ indicating the scores of the lowest 20th percentile in the 80 to 84 year-old group and the lowest 30th percentile in the 85 to 89 year-old group exceeded 180 seconds, some have recommended caution in employing a strict 180 second cut-off. There is ongoing debate in the field of research into the evaluation of fitness-to-drive regarding the optimal cut-off score on the Trails B test.

The objective of this study was to address this controversy by systematically reviewing the evidence for specific Trails B cut-off scores (e.g., cut-offs in both time to completion and number of errors) with respect to fitness-to-drive.

\section{METHODS}

This systematic review was conducted in accordance with the process and methods recommended by the Preferred Reporting Items for Systematic Reviews and Meta-Analyses (PRISMA) guidelines. ${ }^{(9)}$

The need for ethics approval was waived for this study by the Ottawa Hospital Research Ethics Board, as it only involved a literature search.

\section{Literature Search}

An electronic literature search was conducted using CINAHL, Cochrane Database of Systematic Reviews, EMBASE, MEDLINE, PsycINFO, PubMed, and Scopus databases for all relevant English-language publications. No starting date restriction was used in this search. The most updated search was conducted in November 2012. Relevant articles were retrieved using the following subject headings and keywords in various combinations: Trail Making Test, Trail Making Test B, Trail Making B, Trail Making Test Part B, Trail Making Test A and B, Trail Making Test Parts A \& B, Trail Making Test Parts A and B, Trails B, TMT, TMT-B, drive/driving/ driver, auto/automobile, car, vehicle/motor vehicle, accident, traffic, crash, collision, MVA and MVC. This electronic search was supplemented by hand searching of the reference lists of selected articles, meta-analyses, and review articles.

\section{Inclusion and Exclusion Criteria}

All prospective cohort, retrospective cohort, case-control, correlation, and cross-sectional studies reporting the ability of the Trails B test (i.e., the standard Arabic numerals version employing numbers $1-13$ and letters $\mathrm{A}-\mathrm{L}$ ) to predict driving safety were included.

The systematic review was restricted to articles presenting original research findings published in English-language, peerreviewed journals. Reviews, meta-analyses, commentaries, editorials, consensus statements, and guidelines were searched for references, but were not included in the systematic review.

\section{Data Extraction}

Data extraction forms included publication details, investigative site locations, source of participants, design type, sample size, whether power and sample size calculations were provided, age of participants, diseases included (e.g., Alzheimer's Disease, Parkinson's Disease, stroke, traumatic or anoxic brain injury etc.), method of evaluating driving safety (e.g., simulator, on-road, questionnaire, record of crashes), reported associations of Trails B with predicting driving safety, whether a cut-off was reported for Trails B, and source of reported cut-off (study analysis or reference).

Two investigators (MR, FM) independently extracted data from all included studies, and then met to identify and discuss discrepancies in extracted data. Disagreements between the reviewers were discussed and a consensus agreement was reached.

Since Trails B is not routinely employed as part of a multivariate equation in clinical practice, we focused on univariate associations (i.e., the score of the Trails B in isolation, not as part of a multivariate equation).

\section{RESULTS}

Figure 1 illustrates the process of selection of articles for the systematic review. After reviewing 97 articles in detail, including a hand search of the reference sections, a total of 47 articles met the inclusion criteria to be systematically reviewed. Study characteristics are presented in Table 1. The primary outcome (i.e., measures of driving safety) was history of crash (reported or recorded) for $10(21.3 \%)$ studies, simulator test score for $10(21.3 \%)$ studies, and on-road assessment for $27(57.4 \%)$ studies.

Table 2 shows the associations of Trails B with predicting driving safety (primary outcome), organized according to sample sizes in ascending order. Trails B was positively associated with determining fitness-to-drive in 32 out of $47(68.1 \%)$ studies and found to have no association in 15 (31.9\%) studies. 


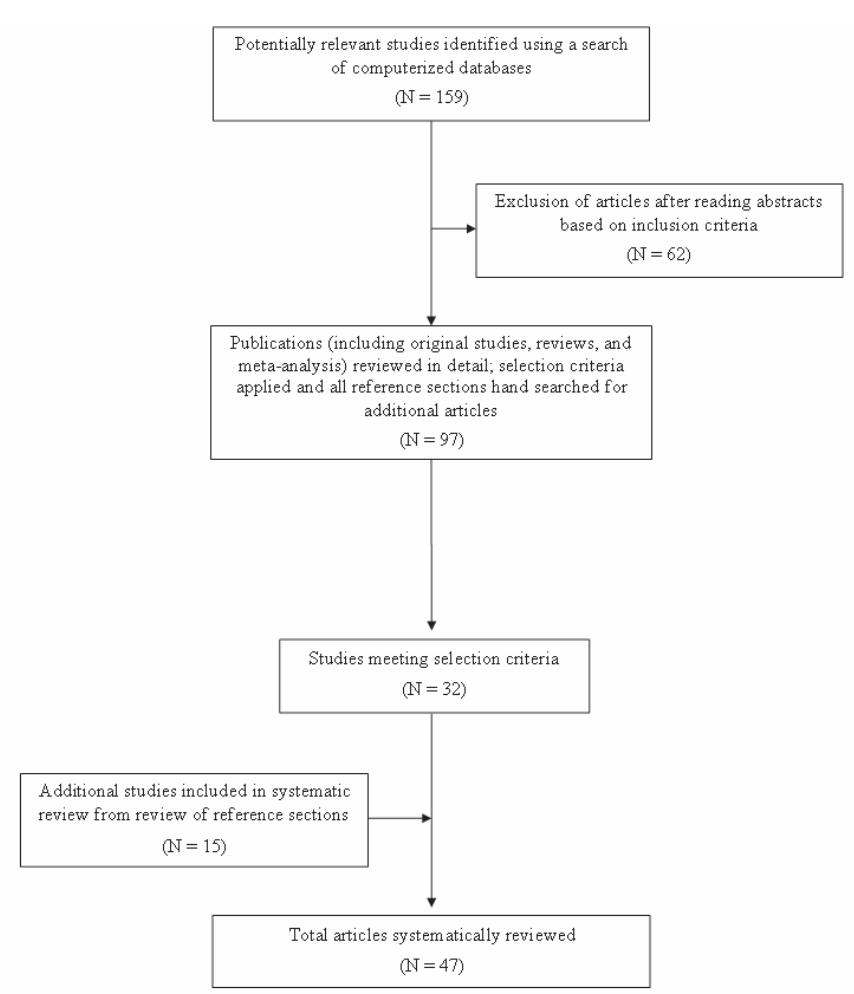

FIGURE 1. Article selection flow diagram

None of the studies justified sample sizes via formal calculations. The sample sizes of many of the studies were small, with 24 (51.1\%) studies having fewer than 100 participants (Table 2). Eleven of these 24 studies with $\mathrm{N}<100 \mathrm{did}$ not find an association of Trails B with driving safety. Stated another way, of the 15 studies showing no association (shaded in gray in Table 2), 11 (73.3\%) had small sizes of $\leq 100$. The remaining four studies with no association had sample sizes of $144,155,176$, and 1,876.

Table 3 shows the studies that reported cut-off values for Trails B in predicting fitness-to-drive. Eight of the 47 studies $(17.0 \%)$ reported cut-off values for Trails B from various sources. Five of these studies reported cut-off values derived from analysis of their data (i.e., primary research): 90 seconds, ${ }^{(10)} 133$ seconds, ${ }^{(11)} 147$ seconds, ${ }^{(12)} 180$ seconds, ${ }^{(13)}$ and $<3$ errors. ${ }^{(14)}$

Three studies reported cut-off values from references cited within their papers: 180 seconds (3 minutes) ${ }^{(15,16)}$ and $\geq 292$ seconds. ${ }^{(17)}$ Two of these references (Table 3 ) are not original research, ${ }^{(18,19)}$ and the remaining three references are not driving studies. ${ }^{6,20,21)}$ The 292 second cut-off was derived from a neuropsychology textbook, ${ }^{(22)}$ not a driving study.

Therefore, in addition to the three continuing medical education articles ${ }^{(2,7,8)}$ recommending a 3 minute or 3 error cut-off (the ' 3 or 3 rule'), this systematic review uncovered four additional articles supporting this cut-off ${ }^{(15,13,16,14)}$ and three other studies recommending even shorter time cut-offs ranging from 90 seconds to 147 seconds. ${ }^{(10,11,12)}$

\section{DISCUSSION}

Some have argued that no in-office tests can determine fitnessto-drive in all situations. This statement is correct, but is often misinterpreted as meaning in-office tests can never be used to determine fitness-to-drive in any situation. While it is obvious that no single in-office tests can be expected to be able to determine fitness-to-drive in all situations, it is a fundamental error in logic to assume therefore that in-office tests cannot determine fitness-to-drive in some situations.

To illustrate the point, as performance on tests such as Trails B progressively worsens with longer completion times and/or more errors, then clinicians should become increasingly comfortable stating a patient "has a potential functional impairment that may increase the risk of crash". For instance, if a patient took 10 minutes to complete Trails B and made ten errors with no concerns regarding the validity of the test, then most physicians would likely feel justified in sending this information to their Ministry of Transportation as a finding that could impact on fitness-to-drive.

The extreme findings described above represent situations in which physicians can determine fitness-to-drive using in-office tests. Situations in which deficits are less glaring are more challenging. One way to address more borderline situations is for physicians to carefully consider precisely what they are being asked to evaluate. In Ontario, Canada, the Highway Traffic Act requires the following:

203. (1) Every legally qualified medical practitioner shall report to the Registrar the name, address and clinical condition of every person sixteen years of age or over attending upon the medical practitioner for medical services who, in the opinion of the medical practitioner, is suffering from a condition that may make it dangerous for the person to operate a motor vehicle. R.S.O. 1990, c. H.8, s. 203. ${ }^{(1)}$

In Ontario, physicians are not asked to determine fitnessto-drive (i.e., they are not asked to report patients as fit or unfit to drive), but rather are asked to report findings that may make it dangerous for the person to drive. The Ministry of Transportation retains responsibility for the final determination of fitness-to-drive. When viewed from this perspective, when selecting Trails B cut-offs that may indicate functional impairment that may impact on fitness-to-drive rather than as a final determination of fitness-to-drive, then Trails B cut-offs of 3 minutes or 3 errors (the ' 3 or 3 rule') remain reasonable to consider when deciding whether or not to bring findings to the attention of the Ministry of Transportation. It is entirely appropriate that the Ministries of Transportation remain responsible for the final determination of fitness-to-drive rather than off-loading their responsibility on MDs. 
ROY:TRAILS B CUT-OFFS IN ASSESSING DRIVING FITNESS

TABLE 1.

Characteristics of included studies

\begin{tabular}{|c|c|c|c|c|c|c|}
\hline & $\begin{array}{c}\text { Author, Year } \\
\text { (Country) }\end{array}$ & $\begin{array}{l}\text { Description of } \\
\text { Study }\end{array}$ & $\begin{array}{l}\text { Description of } \\
\text { Participants }\end{array}$ & Sample Size & $\begin{array}{l}\text { Age in Years } \\
\text { (Mean } \pm S D, \\
\text { Range })\end{array}$ & $\begin{array}{c}\text { Method of } \\
\text { Evaluating } \\
\text { Driving Safety }\end{array}$ \\
\hline \multirow[t]{3}{*}{$\begin{array}{l}\text { Adult Driver (no } \\
\text { age restriction) }\end{array}$} & $\begin{array}{l}\text { Betz, } 2009 \\
(\text { U.S. })^{(15)}\end{array}$ & $\begin{array}{l}\text { A study at a single } \\
\text { Emergency Depart- } \\
\text { ment at a tertiary care } \\
\text { center. TMT B and a } \\
\text { survey of health sta- } \\
\text { tus and driving habits } \\
\text { were administered. } \\
\text { Time to complete } \\
\text { TMT B was com- } \\
\text { pared to published } \\
\text { norms. }\end{array}$ & $\begin{array}{l}\text { A convenience sample } \\
\text { of patients from the } \\
\text { Emergency Depart- } \\
\text { ment. Participants } \\
\text { did not have to be } \\
\text { currently driving to be } \\
\text { included in the study. }\end{array}$ & 144 & $\begin{array}{l}\text { Mean } \\
59 \\
\text { Range } \\
18-95\end{array}$ & $\begin{array}{l}\text { Self-reported } \\
\text { MVCs }\end{array}$ \\
\hline & $\begin{array}{l}\text { Elkin-Frankston, } \\
2007 \text { (U.S.) }^{(23)}\end{array}$ & $\begin{array}{l}\text { A study to examine } \\
\text { the use of the Colour } \\
\text { Trails Tests } 1 \text { and } 2 \text {, } \\
\text { compared to Trails } \\
\text { A and B, in the as- } \\
\text { sessment of driver } \\
\text { competence. }\end{array}$ & $\begin{array}{l}\text { Participants were } \\
\text { recruited through a } \\
\text { driving assessment } \\
\text { program. All partici- } \\
\text { pants were referred for } \\
\text { evaluation of driving } \\
\text { competence by friends, } \\
\text { family members, and } \\
\text { physicians. }\end{array}$ & 29 & $\begin{array}{c}\text { Mean } \\
76.6 \pm 9.5\end{array}$ & $\begin{array}{l}\text { On-road } \\
\text { testing }\end{array}$ \\
\hline & $\begin{array}{c}\text { Niewoehner, } 2012 \\
\text { (U.S.) }^{(30)}\end{array}$ & $\begin{array}{l}\text { A study to develop a } \\
\text { screening battery for } \\
\text { office-based clini- } \\
\text { cians to assist with } \\
\text { deciding who should } \\
\text { proceed to road test- } \\
\text { ing in adults with } \\
\text { cognitive or visual } \\
\text { deficits. }\end{array}$ & $\begin{array}{l}\text { Recruited from a driv- } \\
\text { ing evaluation clinic } \\
\text { at a Veterans Affairs } \\
\text { Medical Centre. }\end{array}$ & 77 & $\begin{array}{l}\text { Mean } \\
67.8 \pm 18.4 \\
\text { Range } \\
23-91\end{array}$ & $\begin{array}{l}\text { On-road } \\
\text { testing }\end{array}$ \\
\hline $\begin{array}{l}\text { Older Driver } \\
\text { (age } \geq 55)\end{array}$ & $\begin{array}{l}\text { Petrakos, } 2009 \\
\left(_{(U . S .)^{(31)}}\right.\end{array}$ & $\begin{array}{l}\text { A study to describe } \\
\text { driving habit charac- } \\
\text { teristics of older driv- } \\
\text { ers referred for formal } \\
\text { driving evaluation } \\
\text { and to compare habits } \\
\text { of drivers found to } \\
\text { be unsafe to drive } \\
\text { with those of safe and } \\
\text { restricted drivers. }\end{array}$ & $\begin{array}{l}\text { A sample from a driv- } \\
\text { ing evaluation clinic } \\
\text { to where subjects had } \\
\text { been referred from } \\
\text { DMV, family physi- } \\
\text { cians, law enforcement } \\
\text { and family members. } \\
\text { All were either cur- } \\
\text { rent drivers or their } \\
\text { licenses were recently } \\
\text { suspended. }\end{array}$ & 57 & $\begin{array}{c}\text { Mean } \\
78.5 \pm 7.0\end{array}$ & $\begin{array}{l}\text { Simulator } \\
\text { score }\end{array}$ \\
\hline
\end{tabular}


ROY:TRAILS B CUT-OFFS IN ASSESSING DRIVING FITNESS

TABLE 1.

Continued

\begin{tabular}{|c|c|c|c|c|c|c|}
\hline & $\begin{array}{c}\text { Author, Year } \\
\text { (Country) }\end{array}$ & $\begin{array}{l}\text { Description of } \\
\text { Study }\end{array}$ & $\begin{array}{c}\text { Description of } \\
\text { Participants }\end{array}$ & Sample Size & $\begin{array}{c}\text { Age in Years } \\
\text { (Mean } \pm S D \\
\text { Range) }\end{array}$ & $\begin{array}{c}\text { Method of } \\
\text { Evaluating } \\
\text { Driving Safety }\end{array}$ \\
\hline \multirow[t]{4}{*}{$\begin{array}{l}\text { Older Driver } \\
\text { (age } \geq 55)\end{array}$} & $\begin{array}{l}\text { Freund, } 2008 \\
(\text { (U.S.) }\end{array}$ & $\begin{array}{l}\text { A study to describe } \\
\text { a population of older } \\
\text { drivers with driving } \\
\text { restrictions, their } \\
\text { most common restric- } \\
\text { tions, and to compare } \\
\text { restricted drivers to } \\
\text { their safe and unsafe } \\
\text { counterparts. }\end{array}$ & $\begin{array}{l}\text { Participants from a } \\
\text { driving clinic referred } \\
\text { by physicians, family, } \\
\text { friends, DMV, or self } \\
\text { referred. All had a } \\
\text { valid driver's license. }\end{array}$ & 108 & $\begin{array}{c}\text { Safe group: } \\
\text { Mean } \\
77.63 \pm 6.62 \\
\text { Range } \\
62-86 \\
\text { Restricted } \\
\text { group: Mean } \\
\text { 78.06 } \pm 8.64 \\
\text { Range } \\
60-99 \\
\\
\text { Unsafe group: } \\
\text { Mean } \\
76.98 \pm 7.60 \\
\text { Range } \\
62-97\end{array}$ & $\begin{array}{l}\text { Simulator } \\
\text { score }\end{array}$ \\
\hline & $\begin{array}{l}\text { Freund, } 2008 \\
\left(_{(U . S .)}\right)^{(33)}\end{array}$ & $\begin{array}{l}\text { A study to assess to } \\
\text { what extent specific } \\
\text { cognitive functions } \\
\text { contribute to pedal } \\
\text { errors among older } \\
\text { drivers. }\end{array}$ & $\begin{array}{l}\text { Participants recruited } \\
\text { through a driving } \\
\text { evaluation clinic, } \\
\text { referred by family } \\
\text { physicians, DMV, or } \\
\text { self referred. All were } \\
\text { currently driving. }\end{array}$ & 176 & $\begin{array}{l}\text { Mean } \\
76 \\
\text { Range } \\
65-89\end{array}$ & $\begin{array}{l}\text { Simulator } \\
\text { score }\end{array}$ \\
\hline & $\begin{array}{l}\text { Wood, } 2008 \text { (Aus- } \\
\text { tralia) }^{(34)}\end{array}$ & $\begin{array}{l}\text { A study to identify a } \\
\text { battery of tests that } \\
\text { predicts safe and } \\
\text { unsafe performance } \\
\text { on an on-road assess- } \\
\text { ment of driving. }\end{array}$ & $\begin{array}{l}\text { Participants were } \\
\text { community-dwelling } \\
\text { individuals } \geq 70 \text { years } \\
\text { old who were living } \\
\text { independently without } \\
\text { walking aids. They } \\
\text { were recruited through } \\
\text { the electoral roll to } \\
\text { participate in a larger } \\
\text { study. Those who were } \\
\text { current drivers were } \\
\text { invited to participate in } \\
\text { this study. }\end{array}$ & 270 & $\begin{array}{c}\text { Mean } \\
75.8 \pm 4.0 \\
\text { Range } \\
70-88\end{array}$ & $\begin{array}{l}\text { On-road test- } \\
\text { ing }\end{array}$ \\
\hline & $\begin{array}{l}\text { Ball, } 2006 \\
\text { (U.S.) }^{(12)}\end{array}$ & $\begin{array}{l}\text { A study to evaluate } \\
\text { the relationship be- } \\
\text { tween performance- } \\
\text { based risk factors and } \\
\text { subsequent, future } \\
\text { at-fault motor vehicle } \\
\text { collision involvement } \\
\text { in a cohort of older } \\
\text { drivers. }\end{array}$ & $\begin{array}{l}\text { Participants were older } \\
\text { adults ( } \geq 55 \text { years old) } \\
\text { presenting to renew } \\
\text { their driver's license at } \\
\text { MVA offices. This is a } \\
\text { similar population to } \\
\text { the MaryPODS study } \\
\text { - see below. }\end{array}$ & 1,910 & $\begin{array}{c}\text { Mean } \\
68.55 \pm 7.95 \\
\text { Range } \\
55-96\end{array}$ & $\begin{array}{l}\text { Database } \\
\text { MVCs }\end{array}$ \\
\hline
\end{tabular}


ROY: TRAILS B CUT-OFFS IN ASSESSING DRIVING FITNESS

TABLE 1.

Continued

\begin{tabular}{|c|c|c|c|c|c|c|}
\hline & $\begin{array}{c}\text { Author, Year } \\
\text { (Country) }\end{array}$ & $\begin{array}{l}\text { Description of } \\
\text { Study }\end{array}$ & $\begin{array}{c}\text { Description of } \\
\text { Participants }\end{array}$ & Sample Size & $\begin{array}{c}\text { Age in Years } \\
\text { (Mean } \pm S D, \\
\text { Range) }\end{array}$ & $\begin{array}{c}\text { Method of } \\
\text { Evaluating } \\
\text { Driving Safety }\end{array}$ \\
\hline \multirow[t]{3}{*}{$\begin{array}{l}\text { Older Driver } \\
\text { (age } \geq 55)\end{array}$} & $\begin{array}{c}\text { Kantor, } 2004 \\
\text { (U.S.) }^{(29)}\end{array}$ & $\begin{array}{l}\text { A study to identify } \\
\text { elements of an older } \\
\text { driver evaluation } \\
\text { program that predict } \\
\text { driving performance } \\
\text { in older adults. }\end{array}$ & $\begin{array}{l}\text { Participants were } \\
\text { referred to the Older } \\
\text { Driver Evaluation Pro- } \\
\text { gram by physicians, } \\
\text { other health profes- } \\
\text { sionals, and family } \\
\text { members. }\end{array}$ & 664 & $\begin{array}{l}\text { No mean, } \\
\text { SD, or range } \\
\text { provided } \\
\text { The only } \\
\text { comment } \\
\text { on age of } \\
\text { participants } \\
\text { was: "65\% of } \\
\text { all participants } \\
\text { were over } \\
\text { age } 70 \text { ". }\end{array}$ & $\begin{array}{l}\text { On-road } \\
\text { testing }\end{array}$ \\
\hline & $\begin{array}{c}\text { Staplin, } 2003 \\
\text { (U.S.) }^{(13) ;} \text { Staplin, } \\
2003 \\
\text { (MaryPODS) }^{(35)}\end{array}$ & $\begin{array}{l}\text { (1) "Model Driver } \\
\text { Screening and Evalu- } \\
\text { ation Program Final } \\
\text { Technical Report, } \\
\text { Maryland Pilot } \\
\text { Older Driver Study } \\
\text { (MaryPODS)": A } \\
\text { study to analyse the } \\
\text { relationships between } \\
\text { functional capac- } \\
\text { ity measures and } \\
\text { future at-fault crash } \\
\text { involvement for older } \\
\text { drivers. The analyses } \\
\text { were based on driving } \\
\text { history data bracket- } \\
\text { ing each individual's } \\
\text { test date by one year } \\
\text { retrospectively, and, } \\
\text { on average, slightly } \\
\text { under } 2 \text { years pro- } \\
\text { spectively. }\end{array}$ & $\begin{array}{c}\text { Participants were } \\
\text { recruited from Motor } \\
\text { Vehicle Administra- } \\
\text { tion (MVA) offices. } \\
\text { All persons age } \geq 55 \\
\text { appearing on random } \\
\text { days for were asked to } \\
\text { volunteer. }\end{array}$ & 1,876 & $\begin{array}{c}\text { Mean } \\
68.28 \pm \_7.92 \\
\text { Range } \\
55-96\end{array}$ & $\begin{array}{l}\text { Database } \\
\text { MVCs }\end{array}$ \\
\hline & & $\begin{array}{l}\text { (2) "MaryPODS } \\
\text { Revisited: Updated } \\
\text { Crash Analysis and } \\
\text { Implications for } \\
\text { Screening Program } \\
\text { Implementation": } \\
\text { Previous analyses was } \\
\text { updated to include } \\
\text { one additional year of } \\
\text { driving experience. }\end{array}$ & $\begin{array}{l}\text { Participants were } \\
\text { recruited from MVA } \\
\text { offices. All persons } \\
\text { age } \geq 55 \text { appearing on } \\
\text { random days for were } \\
\text { asked to volunteer. }\end{array}$ & 1,876 & $\begin{array}{c}\text { Mean } \\
68.28 \pm 7.92 \\
\text { Range } \\
55-96\end{array}$ & $\begin{array}{c}\text { Database } \\
\text { MVCs }\end{array}$ \\
\hline
\end{tabular}


ROY: TRAILS B CUT-OFFS IN ASSESSING DRIVING FITNESS

TABLE 1.

Continued

\begin{tabular}{|c|c|c|c|c|c|c|}
\hline & $\begin{array}{c}\text { Author, Year } \\
\text { (Country) }\end{array}$ & $\begin{array}{l}\text { Description of } \\
\text { Study }\end{array}$ & $\begin{array}{c}\text { Description of } \\
\text { Participants }\end{array}$ & Sample Size & $\begin{array}{c}\text { Age in Years } \\
(\text { Mean } \pm S D \\
\text { Range })\end{array}$ & $\begin{array}{c}\text { Method of } \\
\text { Evaluating } \\
\text { Driving Safety }\end{array}$ \\
\hline \multirow[t]{4}{*}{$\begin{array}{l}\text { Older Driver } \\
\text { (age } \geq 55 \text { ) }\end{array}$} & $\begin{array}{l}\text { Szlyk, } 2002 \\
\left(^{(U . S .)}\right)^{(36)}\end{array}$ & $\begin{array}{l}\text { A study to select a } \\
\text { neuropsychological } \\
\text { battery that correlated } \\
\text { with driving simulator } \\
\text { skills. Administered } \\
\text { MMSE scores served } \\
\text { as a criterion cut-off } \\
\text { for placement into a } \\
\text { group with suspected } \\
\text { dementia or a group } \\
\text { of control subjects. }\end{array}$ & $\begin{array}{l}\text { Participants were } \\
\text { recruited from Dept. } \\
\text { of Veteran Affairs, } \\
\text { memory clinics, and } \\
\text { a geriatric clinic. All } \\
\text { had driving experience } \\
\text { in the past } 2 \text { years. }\end{array}$ & $\begin{array}{c}\mathrm{N}=22 \\
\text { Cases (suspected } \\
\text { dementia) }=8 \\
\text { Controls (normal } \\
\text { cognition) }=14\end{array}$ & $\begin{array}{c}\text { Cases: } \\
\text { Mean } \\
75.6 \pm 7.0 \\
\text { Range } \\
67-85 \\
\text { Controls: } \\
\text { Mean } \\
77.0 \pm 6.2 \\
\text { Range } \\
70-91\end{array}$ & $\begin{array}{l}\text { Simulator } \\
\text { score }\end{array}$ \\
\hline & $\begin{array}{l}\text { Stutts, } 1998 \\
\text { (U.S.) }^{(37)}\end{array}$ & $\begin{array}{l}\text { A study to investi- } \\
\text { gate the usefulness } \\
\text { of } 5 \text { brief tests of } \\
\text { cognitive function for } \\
\text { identifying older driv- } \\
\text { ers who may be at in- } \\
\text { creased risk of crash } \\
\text { involvement. For } \\
\text { each driver, crashes } \\
\text { and convictions were } \\
\text { tallied from a driver } \\
\text { history file over the } \\
\text { 3-year period im- } \\
\text { mediately prior to } \\
\text { license assessment. }\end{array}$ & $\begin{array}{l}\text { All drivers } \geq 65 \text { years } \\
\text { old applying for driv- } \\
\text { er's license renewal } \\
\text { between } 1994-95 \text { were } \\
\text { invited to participate. }\end{array}$ & 3,238 & $\begin{array}{c}\text { Mean } \\
73.6 ; \\
\text { SD and range } \\
\text { not provided }\end{array}$ & $\begin{array}{l}\text { Database } \\
\text { MVCs }\end{array}$ \\
\hline & $\begin{array}{l}\text { Cushman, } 1996 \\
\text { (U.S.) }^{(38)}\end{array}$ & $\begin{array}{l}\text { A study to evaluate } \\
\text { change in drivers' } \\
\text { cognitive abilities } \\
\text { and how this impacts } \\
\text { driver safety by } \\
\text { means of cognitive } \\
\text { testing and on-road } \\
\text { driving evaluations. }\end{array}$ & $\begin{array}{l}\text { Two groups of partici- } \\
\text { pants. The first group } \\
\text { were } 91 \text { drivers over } \\
\text { age } 55 \text { recruited from } \\
\text { the community. The } \\
\text { second group were } \\
32 \text { drivers with early } \\
\text { AD referred from the } \\
\text { Alzheimer Clinic or } \\
\text { Older Adults Clinic. }\end{array}$ & 123 & Not reported & $\begin{array}{l}\text { On-road } \\
\text { testing }\end{array}$ \\
\hline & $\begin{array}{l}\text { Classen, } 2008 \\
\text { (U.S.) }^{(16)}\end{array}$ & $\begin{array}{l}\text { A study to determine } \\
\text { the relationship } \\
\text { between clinical } \\
\text { variables (demo- } \\
\text { graphics, cognitive } \\
\text { testing, comorbidities, } \\
\text { and medications) and } \\
\text { failing a standardized } \\
\text { road test in adults } \\
\text { aged } 65 \text { and older. }\end{array}$ & $\begin{array}{l}\text { Participants were } \\
\text { recruited via advertise- } \\
\text { ments in the com- } \\
\text { munity. There were } 3 \\
\text { waves of recruitments: } \\
\text { one recruiting healthy } \\
\text { older adults, another } \\
\text { recruiting older adults } \\
\text { with multiple comor- } \\
\text { bidities, and a third } \\
\text { recruiting older adults } \\
\text { with movement disor- } \\
\text { ders, specifically PD. }\end{array}$ & 127 & $\begin{array}{c}\text { Mean } \\
74.8 \pm 6.3\end{array}$ & $\begin{array}{l}\text { On-road } \\
\text { testing }\end{array}$ \\
\hline
\end{tabular}


TABLE 1.

Continued

\begin{tabular}{|c|c|c|c|c|c|c|}
\hline & $\begin{array}{c}\text { Author, Year } \\
\text { (Country) }\end{array}$ & $\begin{array}{l}\text { Description of } \\
\text { Study }\end{array}$ & $\begin{array}{c}\text { Description of } \\
\text { Participants }\end{array}$ & Sample Size & $\begin{array}{l}\text { Age in Years } \\
\text { (Mean } \pm S D, \\
\text { Range) }\end{array}$ & $\begin{array}{c}\text { Method of } \\
\text { Evaluating } \\
\text { Driving Safety }\end{array}$ \\
\hline \multirow[t]{5}{*}{$\begin{array}{l}\text { Older Driver } \\
\text { (age } \geq 55)\end{array}$} & $\begin{array}{c}\text { Tarawneh, } 1993 \\
\text { (U.S.) }^{(39)}\end{array}$ & $\begin{array}{l}\text { A 2-year study to } \\
\text { evaluate the correla- } \\
\text { tion between driving } \\
\text { performance and } \\
\text { measured physical } \\
\text { and mental character- } \\
\text { istics of older drivers. }\end{array}$ & $\begin{array}{l}\text { Participants were paid } \\
\text { volunteers who were } \\
\text { active drivers between } \\
\text { the ages of } 65 \text { and } 88 .\end{array}$ & 105 & $\begin{array}{c}\text { Mean } \\
71.4 \\
\text { Range } \\
65-88\end{array}$ & $\begin{array}{l}\text { On-road } \\
\text { testing }\end{array}$ \\
\hline & $\begin{array}{c}\text { Marottoli, } 1998 \\
(\text { (U.S.) })^{(11)}\end{array}$ & $\begin{array}{l}\text { A study to develop } \\
\text { a battery of tests } \\
\text { (visual, cognitive, } \\
\text { and physical) relevant } \\
\text { to driving which } \\
\text { can be performed in } \\
\text { a clinician's office } \\
\text { and to determine } \\
\text { which of these tests } \\
\text { were associated with } \\
\text { self-reported adverse } \\
\text { driving events over } 5 \\
\text { years. }\end{array}$ & $\begin{array}{l}\text { Participants were a } \\
\text { survival cohort from } \\
\text { a previous study, } \\
\text { the Project Safety } \\
\text { cohort, consisting of a } \\
\text { probability sample of } \\
\text { noninstitutionalized, } \\
\text { actively driving indi- } \\
\text { viduals aged } 72 \text { years } \\
\text { and older. }\end{array}$ & 125 & $\begin{array}{c}\text { Mean } \\
81.4 \\
\text { SD and range } \\
\text { not provided }\end{array}$ & Self-report \\
\hline & $\begin{array}{c}\text { Emerson, } 2012 \\
\text { (U.S.) }^{(40)}\end{array}$ & $\begin{array}{l}\text { A study to develop } \\
\text { predictive models } \\
\text { for real-life driving } \\
\text { outcomes in older } \\
\text { drivers. Participants } \\
\text { were followed for } \\
\text { 3-7 years for driving } \\
\text { outcomes. }\end{array}$ & $\begin{array}{l}\text { Healthy volunteers } \\
\text { recruited from the } \\
\text { community via ads and } \\
\text { announcements. }\end{array}$ & 100 & $\begin{array}{l}\text { Mean } \\
72.7 \pm 5.03 \\
\text { Range } \\
65.3-89\end{array}$ & $\begin{array}{c}\text { Self-report } \\
\text { and Database } \\
\text { MVCs }\end{array}$ \\
\hline & 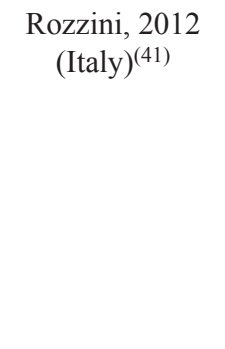 & $\begin{array}{l}\text { A study to examine } \\
\text { the usefulness of spe- } \\
\text { cific neurocognitive } \\
\text { tests for predicting } \\
\text { crash involvement in } \\
\text { participants aged } 80 \\
\text { or older. }\end{array}$ & $\begin{array}{l}\text { Participants were } \\
\text { aged } \geq 80 \text { needing to } \\
\text { renew their licence at } \\
\text { a neuropsychological } \\
\text { clinic. In Italy, neuro- } \\
\text { psychological tests are } \\
\text { required for octogenar- } \\
\text { ians wishing to renew } \\
\text { their licence. }\end{array}$ & 297 & $\begin{array}{c}\text { Mean for } \\
\text { "non-crash } \\
\text { involved" } \\
\text { group }= \\
82.8 \pm 2.8 \\
\text { Mean for "crash } \\
\text { involved" group } \\
=82.6 \pm 3.3\end{array}$ & $\begin{array}{l}\text { Self-report } \\
\\
\end{array}$ \\
\hline & $\begin{array}{l}\text { O'Connor, } 2010 \\
\text { (U.S.) }^{(42)}\end{array}$ & $\begin{array}{l}\text { A study to evaluate } \\
\text { the effectiveness } \\
\text { of an interview- } \\
\text { based screening tool } \\
\text { (including crash his- } \\
\text { tory, family concerns, } \\
\text { clinical condition, and } \\
\text { cognitive function) } \\
\text { in identifying at-risk } \\
\text { older drivers. }\end{array}$ & $\begin{array}{c}\text { Recruited from a clini- } \\
\text { cal driving evaluation } \\
\text { program. }\end{array}$ & 160 & $\begin{array}{l}\text { Mean } 78.3 ; \\
\text { SD and Range } \\
\text { not provided }\end{array}$ & $\begin{array}{l}\text { On-road } \\
\text { testing }\end{array}$ \\
\hline
\end{tabular}


ROY:TRAILS B CUT-OFFS IN ASSESSING DRIVING FITNESS

TABLE 1.

Continued

\begin{tabular}{|c|c|c|c|c|c|c|}
\hline & $\begin{array}{c}\text { Author, Year } \\
\text { (Country) }\end{array}$ & $\begin{array}{l}\text { Description of } \\
\text { Study }\end{array}$ & $\begin{array}{c}\text { Description of } \\
\text { Participants }\end{array}$ & Sample Size & $\begin{array}{c}\text { Age in Years } \\
\text { (Mean } \pm S D \\
\text { Range) }\end{array}$ & $\begin{array}{c}\text { Method of } \\
\text { Evaluating } \\
\text { Driving Safety }\end{array}$ \\
\hline \multirow[t]{2}{*}{$\begin{array}{l}\text { Older Driver } \\
\text { (age } \geq 55)\end{array}$} & $\begin{array}{l}\text { Park, } 2011 \\
\text { (Korea) }^{(43)}\end{array}$ & $\begin{array}{l}\text { A study to find an } \\
\text { association between } \\
\text { cognitive-perceptual } \\
\text { problems of older } \\
\text { drivers and unsafe } \\
\text { driving performance } \\
\text { during driving on a } \\
\text { simulator. }\end{array}$ & $\begin{array}{l}\text { Cases recruited from } \\
\text { a driver evaluation } \\
\text { clinic. Source of con- } \\
\text { trols unclear. }\end{array}$ & $\begin{array}{l}\mathrm{N}=103 \\
\text { Cases (age } \geq 65 \text { ) } \\
=55 \text { Controls } \\
\text { (age late } 20 \text { 's to } \\
\text { early 40's) }=48\end{array}$ & $\begin{array}{c}\text { Cases: } \\
\text { Mean } \\
69.91 \pm 3.63 \\
\text { Controls: } \\
\text { Mean } \\
34.25 \pm 3.62\end{array}$ & $\begin{array}{l}\text { Simulator } \\
\text { score }\end{array}$ \\
\hline & 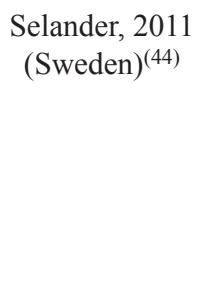 & $\begin{array}{l}\text { A study to investigate } \\
\text { driving errors charac- } \\
\text { teristic for older driv- } \\
\text { ers and relationships } \\
\text { between cognitive } \\
\text { off-road and on-road } \\
\text { test results. }\end{array}$ & $\begin{array}{l}\text { Older drivers (age } \\
65+\text { ) randomly } \\
\text { selected from Vehicle } \\
\text { Registration Office. } \\
\text { Participation volun- } \\
\text { tary. }\end{array}$ & 85 & $\begin{array}{l}\text { Mean } \\
72 \pm 5.3 \\
\text { Range } \\
65-85\end{array}$ & $\begin{array}{l}\text { On-road } \\
\text { testing }\end{array}$ \\
\hline \multirow[t]{2}{*}{$\begin{array}{l}\text { Alzheimer } \\
\text { Disease }\end{array}$} & $\begin{array}{c}\text { Dawson, } 2009 \\
\text { (U.S.) }^{(45)}\end{array}$ & $\begin{array}{l}\text { A study to measure } \\
\text { the association of } \\
\text { cognition, visual } \\
\text { perception, and motor } \\
\text { function with driving } \\
\text { safety in AD. }\end{array}$ & $\begin{array}{l}\text { AD patients were } \\
\text { recruited from a } \\
\text { registry maintained } \\
\text { by the Dept. of } \\
\text { Neurology. Controls } \\
\text { were volunteers in } \\
\text { the local community, } \\
\text { with no neurological } \\
\text { diagnosis or } \\
\text { complaints and no } \\
\text { personal or family } \\
\text { report of abnormal } \\
\text { cognitive decline. All } \\
\text { were active drivers. }\end{array}$ & $\begin{array}{c}\mathrm{N}=155 \\
\text { Cases (probable } \\
\text { early dementia) } \\
=40 \\
\text { Controls } \\
\text { (neurologically } \\
\text { normal) }=115\end{array}$ & $\begin{array}{l}\text { Cases: } \\
\text { Mean } \\
75.1 \pm 7.7 \\
\text { Controls: } \\
\text { Mean } \\
69.4 \pm 7.0\end{array}$ & $\begin{array}{l}\text { On-road } \\
\text { testing }\end{array}$ \\
\hline & $\begin{array}{c}\text { Grace, } 2005 \\
\text { (U.S.) }^{(46)}\end{array}$ & $\begin{array}{l}\text { A study to examine } \\
\text { neuropsychological } \\
\text { and motor deficits } \\
\text { in PD that may } \\
\text { contribute to } \\
\text { driving impairment, } \\
\text { comparing patients } \\
\text { with PD to patients } \\
\text { with AD and to } \\
\text { healthy elderly } \\
\text { controls. }\end{array}$ & $\begin{array}{l}\text { PD patients were } \\
\text { drawn consecutively } \\
\text { from a hospital-based } \\
\text { movement disorders } \\
\text { clinic. AD patients } \\
\text { were recruited through } \\
\text { a hospital-based } \\
\text { memory disorders } \\
\text { clinic. Control } \\
\text { subjects were age } \\
\text { and education } \\
\text { matched community } \\
\text { volunteers or } \\
\text { nondemented spouses } \\
\text { of AD patients. All } \\
\text { participants were } \\
\text { currently driving. }\end{array}$ & $\begin{array}{c}\mathrm{N}=62 \\
\mathrm{PD} \text { group }=21 \mathrm{AD} \\
\text { group }=20 \\
\text { Controls }=21\end{array}$ & $\begin{array}{c}\text { PD: Mean } \\
68.1 \pm 8.5 \\
\text { Range } \\
45-83 \\
\text { AD: Mean } \\
70.8 \pm 7.1 \\
\text { Range } \\
\text { 59-85 } \\
\text { Controls: Mean } \\
\text { 69.0 } \pm 10.4 \\
\text { Range } \\
\text { 46-85 }\end{array}$ & $\begin{array}{l}\text { On-road } \\
\text { testing }\end{array}$ \\
\hline
\end{tabular}


ROY: TRAILS B CUT-OFFS IN ASSESSING DRIVING FITNESS

TABLE 1.

Continued

\begin{tabular}{|c|c|c|c|c|c|c|}
\hline & $\begin{array}{c}\text { Author, Year } \\
\text { (Country) }\end{array}$ & $\begin{array}{l}\text { Description of } \\
\text { Study }\end{array}$ & $\begin{array}{c}\text { Description of } \\
\text { Participants }\end{array}$ & Sample Size & $\begin{array}{c}\text { Age in Years } \\
\text { (Mean } \pm S D, \\
\text { Range) }\end{array}$ & $\begin{array}{c}\text { Method of } \\
\text { Evaluating } \\
\text { Driving Safety }\end{array}$ \\
\hline \multirow[t]{3}{*}{$\begin{array}{l}\text { Alzheimer } \\
\text { Disease }\end{array}$} & $\begin{array}{c}\text { Rizzo, } 1997 \\
\text { (U.S.) }^{(47)}\end{array}$ & $\begin{array}{l}\text { A study to examine } \\
\text { the effect of AD } \\
\text { on driver collision } \\
\text { avoidance using a } \\
\text { driving simulator, } \\
\text { and how these } \\
\text { unsafe events are } \\
\text { predicted by visual } \\
\text { and cognitive factors } \\
\text { sensitive to decline in } \\
\text { aging and AD. }\end{array}$ & $\begin{array}{l}\text { AD patients were } \\
\text { recruited from a } \\
\text { registry in the AD } \\
\text { Research Center of the } \\
\text { Dept. of Neurology. } \\
\text { Control subjects } \\
\text { were volunteers in } \\
\text { the local community. } \\
\text { All participants held } \\
\text { a current driver's } \\
\text { license, although some } \\
\text { had reduced driving } \\
\text { activity due to self } \\
\text { or family-imposed } \\
\text { restrictions. }\end{array}$ & $\begin{array}{c}\mathrm{N}=39 \\
\text { AD group }=21 \\
\text { Controls }=18\end{array}$ & $\begin{array}{l}\text { AD: Mean } \\
71.5 \pm 8.5 \\
\text { Controls: } \\
\text { Mean } \\
71.9 \pm 5.5\end{array}$ & $\begin{array}{l}\text { Simulator } \\
\text { score }\end{array}$ \\
\hline & $\begin{array}{c}\text { Fox, } 1997 \\
\text { (Australia) }^{(48)}\end{array}$ & $\begin{array}{l}\text { A study to examine } \\
\text { driving competence } \\
\text { in drivers diagnosed } \\
\text { with probable AD } \\
\text { using on-road testing } \\
\text { and to examine } \\
\text { the validity of a } \\
\text { standardized medical } \\
\text { exam, MMSE, and } \\
\text { neuropsychological } \\
\text { assessment as } \\
\text { predictors of } \\
\text { open road driving } \\
\text { performance. }\end{array}$ & $\begin{array}{c}\text { Subjects had a } \\
\text { diagnosis of probable } \\
\text { AD and were } \\
\text { consecutively referred } \\
\text { for driver assessment } \\
\text { from specialist } \\
\text { Dementia Clinics. All } \\
\text { subjects, except one, } \\
\text { were still driving. }\end{array}$ & 19 & $\begin{array}{c}\text { Mean } \\
74.3 \pm 6.4 \\
\text { Range } \\
59-84\end{array}$ & $\begin{array}{l}\text { On-road } \\
\text { testing }\end{array}$ \\
\hline & $\begin{array}{c}\text { Rizzo, } 2001 \\
\text { (U.S.) }^{(49)}\end{array}$ & $\begin{array}{l}\text { A study to test } \\
\text { whether drivers with } \\
\text { mild to moderate AD } \\
\text { are at greater risk for } \\
\text { intersection crashes } \\
\text { compared to normal } \\
\text { controls. }\end{array}$ & $\begin{array}{l}\text { AD patients were } \\
\text { recruited from a } \\
\text { registry in the AD } \\
\text { Research Center. } \\
\text { Control subjects were } \\
\text { volunteers in the local } \\
\text { community. All held a } \\
\text { valid driver's licence, } \\
\text { although some had } \\
\text { reduced driving due to } \\
\text { self or family-imposed } \\
\text { restrictions. }\end{array}$ & $\begin{array}{c}\mathrm{N}=30 \\
\mathrm{AD} \text { group }=18 \\
\text { Controls }=12\end{array}$ & $\begin{array}{c}\text { AD: Mean } \\
73 \pm 7 \\
\text { Controls: } \\
\text { Mean } \\
70 \pm 4.7\end{array}$ & $\begin{array}{l}\text { Simulator } \\
\text { score }\end{array}$ \\
\hline
\end{tabular}


ROY: TRAILS B CUT-OFFS IN ASSESSING DRIVING FITNESS

TABLE 1 .

Continued

\begin{tabular}{|c|c|c|c|c|c|c|}
\hline & $\begin{array}{c}\text { Author, Year } \\
\text { (Country) }\end{array}$ & $\begin{array}{l}\text { Description of } \\
\text { Study }\end{array}$ & $\begin{array}{c}\text { Description of } \\
\text { Participants }\end{array}$ & Sample Size & $\begin{array}{c}\text { Age in Years } \\
\text { (Mean } \pm S D \\
\text { Range) }\end{array}$ & $\begin{array}{c}\text { Method of } \\
\text { Evaluating } \\
\text { Driving Safety }\end{array}$ \\
\hline \multirow[t]{2}{*}{$\begin{array}{l}\text { Alzheimer } \\
\text { Disease }\end{array}$} & $\begin{array}{l}\text { Ott, } 2003 \\
\left(^{(U . S .}\right)^{(50)}\end{array}$ & $\begin{array}{l}\text { A study to compare } \\
\text { a 4-point caregiver } \\
\text { rating scale of } \\
\text { driving ability to a } \\
\text { battery of standard } \\
\text { neuropsychological } \\
\text { tests given to subjects } \\
\text { with questionable } \\
\text { or mild dementia. } \\
\text { Based on the results } \\
\text { of Part A, a follow up } \\
\text { study (Part B) was } \\
\text { conducted with only } \\
\text { Proteus Mazes in } \\
\text { normal subjects and } \\
\text { those with mild to } \\
\text { moderate dementia. } \\
\text { Only Part A of this } \\
\text { study contained Trails } \\
\text { B, so only Part A } \\
\text { methods and results } \\
\text { will be presented } \\
\text { in this systematic } \\
\text { review. }\end{array}$ & $\begin{array}{l}\text { In Part A, patients } \\
\text { were drawn from a } \\
\text { Memory Disorders } \\
\text { Clinic. All had } \\
\text { probable AD by } \\
\text { NINCDS-ADRDA } \\
\text { criteria. In Part B, } \\
\text { subjects were drawn } \\
\text { from another Memory } \\
\text { Disorders Clinic, and } \\
\text { this sample consisted } \\
\text { of both normal } \\
\text { subjects and those } \\
\text { with mild-moderate } \\
\text { dementia based on } \\
\text { CDR criteria. }\end{array}$ & 27 & $\begin{array}{c}\text { Mean } \\
74.8 \pm 5.9\end{array}$ & $\begin{array}{l}\text { Four-point } \\
\text { driving ability } \\
\text { rating scale } \\
\text { completed } \\
\text { by caregiver } \\
\text { or family } \\
\text { member. }\end{array}$ \\
\hline & $\begin{array}{l}\text { Uc, } 2005 \\
\left(^{(U . S .}\right)^{(51)}\end{array}$ & $\begin{array}{l}\text { A study to assess } \\
\text { visual search and } \\
\text { recognition of } \\
\text { roadside targets and } \\
\text { safety errors during a } \\
\text { landmark and traffic } \\
\text { sign identification } \\
\text { task in driver with } \\
\text { AD compared to } \\
\text { neurologically normal } \\
\text { older adults. }\end{array}$ & $\begin{array}{l}\text { Participants with mild } \\
\text { AD consistent with } \\
\text { NINCDS-ADRDA } \\
\text { criteria were recruited } \\
\text { from a registry in the } \\
\text { Dept. of Neurology. } \\
\text { Controls were } \\
\text { volunteers in the } \\
\text { local community. All } \\
\text { participants were still } \\
\text { driving, although some } \\
\text { had reduced driving } \\
\text { activity due to self } \\
\text { or family-imposed } \\
\text { restrictions. }\end{array}$ & $\begin{array}{c}\mathrm{N}=170 \\
\mathrm{AD} \text { group }=33 \\
\text { Controls }=137\end{array}$ & $\begin{array}{c}\text { AD: Mean } \\
76.1 \pm 6.3 \\
\text { Controls: Mean } \\
64.3 \pm 11.4\end{array}$ & $\begin{array}{l}\text { On-road } \\
\text { testing }\end{array}$ \\
\hline
\end{tabular}


ROY: TRAILS B CUT-OFFS IN ASSESSING DRIVING FITNESS

TABLE 1.

Continued

\begin{tabular}{|c|c|c|c|c|c|c|}
\hline & $\begin{array}{c}\text { Author, Year } \\
\text { (Country) }\end{array}$ & $\begin{array}{l}\text { Description of } \\
\text { Study }\end{array}$ & $\begin{array}{c}\text { Description of } \\
\text { Participants }\end{array}$ & Sample Size & $\begin{array}{c}\text { Age in Years } \\
\text { (Mean } \pm S D \\
\text { Range })\end{array}$ & $\begin{array}{c}\text { Method of } \\
\text { Evaluating } \\
\text { Driving Safety }\end{array}$ \\
\hline \multirow[t]{2}{*}{$\begin{array}{l}\text { Alzheimer } \\
\text { Disease }\end{array}$} & Uc, 2006 (U.S.) $)^{(52)}$ & $\begin{array}{l}\text { A study to test } \\
\text { rear-end collision } \\
\text { avoidance in mild } \\
\text { AD compared with } \\
\text { elderly controls using } \\
\text { a driving simulator. }\end{array}$ & $\begin{array}{l}\text { Subjects with mild AD } \\
\text { (based on NINCDS- } \\
\text { ADRDA criteria) } \\
\text { were recruited from } \\
\text { a registry in the } \\
\text { Dept. of Neurology. } \\
\text { Control participants } \\
\text { were neurologically } \\
\text { normal adults } \\
\text { volunteering from the } \\
\text { local community. All } \\
\text { were active drivers, } \\
\text { although AD subjects } \\
\text { reported significantly } \\
\text { less driving activity } \\
\text { due to self or family- } \\
\text { imposed restrictions. }\end{array}$ & $\begin{array}{c}\mathrm{N}=176 \\
\text { Cases }=61 \\
\text { Controls }=115\end{array}$ & $\begin{array}{c}\text { Cases: Mean } \\
73.5 \pm 8.5 \\
\text { Controls: } \\
69.4 \pm 6.7\end{array}$ & $\begin{array}{l}\text { Simulator } \\
\text { score }\end{array}$ \\
\hline & $\begin{array}{l}\text { Ott, } 2008 \\
(\text { U.S. })^{(53)}\end{array}$ & $\begin{array}{l}\text { A study to examine } \\
\text { the ability of } \\
\text { computerized maze } \\
\text { test performance } \\
\text { to predict road test } \\
\text { performance of } \\
\text { cognitively impaired } \\
\text { and normal older } \\
\text { drivers. }\end{array}$ & $\begin{array}{l}\text { Cases recruited from a } \\
\text { Memory Assessment } \\
\text { Program and a } \\
\text { Memory Disorders } \\
\text { Center. Controls } \\
\text { recruited from } \\
\text { participants' family } \\
\text { and friends. }\end{array}$ & $\begin{array}{c}\mathrm{N}=121 \\
\text { Cases (probable or } \\
\text { possible AD) } \\
=76 \\
\text { Controls } \\
\text { (without cognitive } \\
\text { impairment) }=45\end{array}$ & $\begin{array}{l}\text { Cases: } \\
\text { Mean } \\
75.8 \pm 6.9 \\
\text { Controls: } \\
\text { Mean } \\
73.6 \pm 9\end{array}$ & $\begin{array}{l}\text { On-road } \\
\text { testing }\end{array}$ \\
\hline $\begin{array}{l}\text { Parkinson's } \\
\text { Disease }\end{array}$ & Uc, 2006 (U.S.) $)^{(25)}$ & $\begin{array}{l}\text { A study to assess } \\
\text { the ability for } \\
\text { visual search and } \\
\text { recognition of } \\
\text { roadside targets and } \\
\text { safety errors during a } \\
\text { landmark and traffic } \\
\text { sign identification } \\
\text { task in drivers with } \\
\text { PD. }\end{array}$ & $\begin{array}{c}\text { Patients with mild } \\
\text { to moderate PD } \\
\text { were recruited } \\
\text { from Movement } \\
\text { Disorders clinics. } \\
\text { Control subjects } \\
\text { were neurologically } \\
\text { normal elderly adults. } \\
\text { All participants were } \\
\text { community-dwelling, } \\
\text { independently living, } \\
\text { and licensed active } \\
\text { drivers. }\end{array}$ & $\begin{array}{c}\mathrm{N}=230 \\
\text { Cases (mild to } \\
\text { moderate PD) } \\
=79 \\
\text { Controls } \\
\text { (neurologically } \\
\text { normal elderly } \\
\text { adults) }=151\end{array}$ & $\begin{array}{c}\text { Cases: } \\
\text { Mean } \\
65.9 \pm 8.6 \\
\text { Controls: } \\
65.3 \pm 11.5\end{array}$ & $\begin{array}{l}\text { On-road } \\
\text { testing }\end{array}$ \\
\hline
\end{tabular}


TABLE 1.

Continued

\begin{tabular}{|c|c|c|c|c|c|c|}
\hline & $\begin{array}{c}\text { Author, Year } \\
\text { (Country) }\end{array}$ & $\begin{array}{l}\text { Description of } \\
\text { Study }\end{array}$ & $\begin{array}{l}\text { Description of } \\
\text { Participants }\end{array}$ & Sample Size & $\begin{array}{c}\text { Age in Years } \\
\text { (Mean } \pm S D \\
\text { Range) }\end{array}$ & $\begin{array}{c}\text { Method of } \\
\text { Evaluating } \\
\text { Driving Safety }\end{array}$ \\
\hline \multirow[t]{2}{*}{$\begin{array}{l}\text { Parkinson's } \\
\text { Disease }\end{array}$} & Grace, $\underset{(46)}{2005}$ (U.S.) & $\begin{array}{l}\text { A study to examine } \\
\text { neuropsychological } \\
\text { and motor deficits } \\
\text { in PD that may } \\
\text { contribute to } \\
\text { driving impairment, } \\
\text { comparing patients } \\
\text { with PD to patients } \\
\text { with AD and to } \\
\text { healthy elderly } \\
\text { controls. }\end{array}$ & $\begin{array}{l}\text { PD patients were } \\
\text { drawn consecutively } \\
\text { forma hospital-based } \\
\text { movement disorders } \\
\text { clinic. AD patients } \\
\text { were recruited through } \\
\text { a hospital-based } \\
\text { memory disorders } \\
\text { clinic. Control } \\
\text { subjects were age } \\
\text { and education } \\
\text { matched community } \\
\text { volunteers or } \\
\text { nondemented spouses } \\
\text { of AD patients. All } \\
\text { participants were } \\
\text { currently driving. }\end{array}$ & $\begin{array}{c}\mathrm{N}=62 \\
\mathrm{PD} \text { group }=21 \\
\text { AD group }=20 \\
\text { Controls }=21\end{array}$ & $\begin{array}{l}\text { PD: Mean } \\
68.1 \pm 8.5 \\
\text { Range } \\
45-83 \\
\text { AD: Mean } \\
70.8 \pm 7.1 \\
\text { Range } \\
\text { 59-85 } \\
\text { Controls: Mean } \\
\text { 69.0 } \pm 10.4 \\
\text { Range } \\
\text { 46-85 }\end{array}$ & $\begin{array}{l}\text { On-road } \\
\text { testing }\end{array}$ \\
\hline & $\begin{array}{l}\text { Scally, } 2011 \\
\text { (Australia) }^{(26)}\end{array}$ & $\begin{array}{l}\text { A study to investigate } \\
\text { the impact of external } \\
\text { cue validity on } \\
\text { simulated driving } \\
\text { performance in PD } \\
\text { compared to controls. }\end{array}$ & $\begin{array}{l}\text { Cases were drivers } \\
\text { with PD diagnosed by } \\
\text { a neurologist. Source } \\
\text { of cases and controls } \\
\text { not explicitly stated. }\end{array}$ & $\begin{array}{c}\mathrm{N}=28 \\
\text { Cases (with PD) } \\
=19 \text { Controls } \\
\text { (healthy, age- } \\
\text { matched) }=19\end{array}$ & $\begin{array}{c}\text { Cases: Mean } \\
68.74 \pm 6.72 \\
\text { Range } 52-81 \\
\text { Controls: Mean } \\
68.05 \pm 7.2 \\
\text { Range } 56-78\end{array}$ & $\begin{array}{l}\text { Simulator } \\
\text { score }\end{array}$ \\
\hline $\begin{array}{l}\text { Dementia, } \\
\text { not specified }\end{array}$ & $\begin{array}{l}\text { Carr, } 2011 \\
\text { (U.S.) }^{(54)}\end{array}$ & $\begin{array}{l}\text { A study to develop } \\
\text { a cognitive and } \\
\text { functional screening } \\
\text { battery for the on- } \\
\text { road performance of } \\
\text { older drivers with } \\
\text { dementia. }\end{array}$ & $\begin{array}{l}\text { Recruited from a } \\
\text { driving evaluation } \\
\text { clinic. Participants } \\
\text { had a diagnosis } \\
\text { of dementia from } \\
\text { physician referral or } \\
\text { from AD-8 (Aging } \\
\text { and Dementia-8) } \\
\text { questionnaire } \\
\text { completed by an } \\
\text { informant. }\end{array}$ & 85 & $\begin{array}{c}\text { Mean } \\
74.2 \pm 9 \\
\text { Range } \\
52-90\end{array}$ & $\begin{array}{l}\text { On-road } \\
\text { testing }\end{array}$ \\
\hline $\begin{array}{l}\text { Questionable } \\
\text { Dementia } \\
\text { (CDR }=0.5) \\
\text { including } \\
\text { possible AD, } \\
\text { stroke, remote } \\
\text { history of alcohol } \\
\text { abuse, or head } \\
\text { trauma }\end{array}$ & $\begin{array}{l}\text { Whelihan, } 2005 \\
\text { (U.S.) }\end{array}$ & $\begin{array}{l}\text { A study to investigate } \\
\text { the role of visual } \\
\text { attention and } \\
\text { executive measures } \\
\text { in predicting driving } \\
\text { competence in older } \\
\text { individuals with } \\
\text { early-stage cognitive } \\
\text { decline compared to } \\
\text { age-matched controls. }\end{array}$ & $\begin{array}{l}\text { Participants in the } \\
\text { patient group all } \\
\text { had a CDR of } 0.5 \\
\text { and were recruited } \\
\text { sequentially from a } \\
\text { Memory Disorders } \\
\text { Clinic. Controls } \\
\text { all had a CDR of 0 } \\
\text { (cognitively intact) and } \\
\text { were recruited from } \\
\text { the local community } \\
\text { via ads. }\end{array}$ & $\begin{array}{c}\mathrm{N}=46 \\
\text { Questionable } \\
\text { dementia group } \\
(\text { CDR } 0.5)=23 \\
\text { Controls }(\text { CDR } 0) \\
=23\end{array}$ & $\begin{array}{l}\text { Cases: } \\
\text { Mean } \\
78.2 \pm 9.3 \\
\text { Controls: } \\
\text { Mean } \\
74.3 \pm 7.3\end{array}$ & $\begin{array}{l}\text { On-road } \\
\text { testing }\end{array}$ \\
\hline
\end{tabular}


TABLE 1.

Continued

\begin{tabular}{|c|c|c|c|c|c|c|}
\hline & $\begin{array}{c}\text { Author, Year } \\
\text { (Country) }\end{array}$ & $\begin{array}{l}\text { Description of } \\
\text { Study }\end{array}$ & $\begin{array}{l}\text { Description of } \\
\text { Participants }\end{array}$ & Sample Size & $\begin{array}{l}\text { Age in Years } \\
\text { (Mean } \pm S D, \\
\text { Range) }\end{array}$ & $\begin{array}{c}\text { Method of } \\
\text { Evaluating } \\
\text { Driving Safety }\end{array}$ \\
\hline \multirow{5}{*}{$\begin{array}{l}\text { Acquired } \\
\text { Cognitive } \\
\text { Impairment } \\
\text { after Traumatic } \\
\text { Brain Injury, } \\
\text { Stroke, } \\
\text { Hemorrhage, } \\
\text { Encephalitis, } \\
\text { Tumour, or other } \\
\text { CNS disorders } \\
\text { (e.g., Multiple } \\
\text { Sclerosis, } \\
\text { Huntington } \\
\text { Disease) }\end{array}$} & $\begin{array}{l}\text { Alexandersen, } \\
2009 \text { (Norway) }^{(56)}\end{array}$ & $\begin{array}{l}\text { A study to investigate } \\
\text { the predictive value } \\
\text { of neuropsychological } \\
\text { tests for on-road } \\
\text { evaluation outcome } \\
\text { after inconclusive } \\
\text { assessment. }\end{array}$ & $\begin{array}{l}\text { Outpatients at Dept. } \\
\text { of Physical Medicine } \\
\text { and Rehabilitation } \\
\text { referred for evaluation } \\
\text { of fitness to drive } \\
\text { after inconclusive } \\
\text { neuropsychological } \\
\text { assessment. }\end{array}$ & 35 & $\begin{array}{c}\text { Mean } \\
47.4 \pm 13.7\end{array}$ & $\begin{array}{l}\text { On-road } \\
\text { testing }\end{array}$ \\
\hline & $\begin{array}{l}\text { Lundqvist, } 2007 \\
\text { (Sweden) }^{(57)}\end{array}$ & $\begin{array}{l}\text { A study to assess } \\
\text { drivers with acquired } \\
\text { brain injury on } \\
\text { cognitive functions, } \\
\text { driving performance, } \\
\text { and the drivers'self- } \\
\text { rating of their driving. }\end{array}$ & $\begin{array}{l}\text { The participants } \\
\text { were a consecutive } \\
\text { sample of patients } \\
\text { with brain injury who } \\
\text { received outpatient } \\
\text { rehabilitation } \\
\text { services at the Dept. } \\
\text { of Rehabilitation } \\
\text { Medicine. }\end{array}$ & 30 & $\begin{array}{c}\text { Mean } \\
51.6 \pm 11.21 \\
\text { Range } \\
21-75\end{array}$ & $\begin{array}{l}\text { On-road } \\
\text { testing }\end{array}$ \\
\hline & $\begin{array}{l}\text { Mazer, } 1998 \\
{\text { (Canada })^{(14)}}^{(\text {Can }}\end{array}$ & $\begin{array}{l}\text { A study to determine } \\
\text { the ability of } \\
\text { perceptual testing } \\
\text { to predict on-road } \\
\text { driving outcome in } \\
\text { subjects with stroke. }\end{array}$ & $\begin{array}{c}\text { Subjects with } \\
\text { stroke referred to a } \\
\text { Driving Evaluation } \\
\text { Service, including } \\
\text { both inpatients at } \\
\text { a Rehabilitation } \\
\text { Hospital and outpatient } \\
\text { referrals. }\end{array}$ & 84 & $\begin{array}{l}\text { Mean } \\
60.8 \pm 11.9 \\
\text { Range } \\
27-84\end{array}$ & $\begin{array}{l}\text { On-road } \\
\text { testing }\end{array}$ \\
\hline & $\begin{array}{l}\text { Devos, } 2012 \\
\text { (Belgium) }^{(58)}\end{array}$ & $\begin{array}{l}\text { A study to identify the } \\
\text { most accurate clinical } \\
\text { predictors of fitness to } \\
\text { drive in HD. }\end{array}$ & $\begin{array}{l}\text { Cases were all active } \\
\text { drivers recruited } \\
\text { from HD clinic at a } \\
\text { university hospital. } \\
\text { Source of controls not } \\
\text { clear. }\end{array}$ & $\begin{array}{c}\mathrm{N}=60 \\
\text { Cases (with HD) }= \\
30 \text { Healthy controls } \\
=30\end{array}$ & $\begin{array}{c}\text { Cases: } \\
\text { Mean } \\
50.2 \pm 12.4 \\
\text { Controls: } \\
\text { Mean } \\
50.26 \pm 12.64\end{array}$ & $\begin{array}{l}\text { On-road } \\
\text { testing }\end{array}$ \\
\hline & $\begin{array}{l}\text { Bliokas, } 2011 \\
\text { (Australia) }^{(17)}\end{array}$ & $\begin{array}{c}\text { A study to evaluate a } \\
\text { neuropsychological } \\
\text { assessment battery } \\
\text { and its individual test } \\
\text { components to assess } \\
\text { fitness to drive in } \\
\text { cognitively impaired } \\
\text { individuals (including } \\
\text { traumatic brain } \\
\text { injury, stroke, PD, } \\
\text { dementia). }\end{array}$ & $\begin{array}{l}\text { Participants were } \\
\text { referred for driving } \\
\text { assessment after } \\
\text { neurological injury to } \\
\text { a Brain Injury Service } \\
\text { and Rehab Unit. }\end{array}$ & 104 & $\begin{array}{c}\text { Mean } \\
61.35 \pm 16.71 \\
\text { Range } \\
17-93\end{array}$ & $\begin{array}{l}\text { On-road } \\
\text { testing }\end{array}$ \\
\hline
\end{tabular}


TABLE 1.

Continued

\begin{tabular}{|c|c|c|c|c|c|c|}
\hline & $\begin{array}{c}\text { Author, Year } \\
\text { (Country) }\end{array}$ & $\begin{array}{l}\text { Description of } \\
\text { Study }\end{array}$ & $\begin{array}{l}\text { Description of } \\
\text { Participants }\end{array}$ & Sample Size & $\begin{array}{l}\text { Age in Years } \\
(\text { Mean } \pm S D, \\
\text { Range })\end{array}$ & $\begin{array}{c}\text { Method of } \\
\text { Evaluating } \\
\text { Driving Safety }\end{array}$ \\
\hline $\begin{array}{l}\text { Acquired } \\
\text { Cognitive } \\
\text { Impairment } \\
\text { after Traumatic } \\
\text { Brain Injury, } \\
\text { Stroke, } \\
\text { Hemorrhage, } \\
\text { Encephalitis, } \\
\text { Tumour, or other } \\
\text { CNS disorders } \\
\text { (e.g., Multiple } \\
\text { Sclerosis, } \\
\text { Huntington } \\
\text { Disease) }\end{array}$ & $\begin{array}{l}\text { Soderstrom, } 2006 \\
\text { (Sweden) }^{(59)}\end{array}$ & $\begin{array}{l}\text { A study to examine } \\
\text { the predictive value of } \\
\text { a neuropsychological } \\
\text { test battery relating } \\
\text { to an on-road driving } \\
\text { evaluation in patients } \\
\text { with stroke and to } \\
\text { determine whether } \\
\text { patients who failed } \\
\text { the evaluation could } \\
\text { improve their driving } \\
\text { through behind-the- } \\
\text { wheel training. }\end{array}$ & $\begin{array}{l}\text { Cases were patients } \\
\text { admitted consecutively } \\
\text { to hospital for stroke. } \\
\text { All had valid licence. } \\
\text { Interval between } \\
\text { stroke onset and } \\
\text { examination ranged } \\
\text { from } 1.4 \text { to } 14 \text { months. } \\
\text { Healthy controls were } \\
\text { recruited via ad in } \\
\text { newspaper. }\end{array}$ & $\begin{array}{c}\mathrm{N}=54 \\
\text { Cases } \\
(\text { with stroke) }=34 \\
\text { Controls }=20\end{array}$ & $\begin{array}{c}\text { Range for all } \\
\text { subjects }= \\
25-67 \\
\text { Cases: Mean } \\
54 \pm 8.8 \\
\text { Controls: Mean } \\
\text { and SD not } \\
\text { reported }\end{array}$ & $\begin{array}{l}\text { On-road } \\
\text { testing }\end{array}$ \\
\hline \multirow[t]{3}{*}{$\begin{array}{l}\text { Acquired } \\
\text { Brain Injury } \\
\text { (Traumatic } \\
\text { Brain Injury, } \\
\text { Anoxic Brain } \\
\text { Injury, Stroke) }\end{array}$} & $\begin{array}{l}\text { Hartman-Maier, } \\
2008 \text { (Israel) }^{(24)}\end{array}$ & $\begin{array}{l}\text { A study to examine } \\
\text { the validity of the } \\
\text { Colour Trails Test } \\
\text { in the pre-driver } \\
\text { assessment of } \\
\text { individuals with } \\
\text { acquired brain injury } \\
\text { (including traumatic } \\
\text { brain injury, anoxic } \\
\text { brain injury, stroke). }\end{array}$ & $\begin{array}{l}\text { Participants with } \\
\text { acquired brain injury } \\
\text { were selected from } \\
\text { a pool of clients } \\
\text { referred to a driving } \\
\text { rehabilitation } \\
\text { program within the } \\
\text { Occupational Therapy } \\
\text { Dept. at a central } \\
\text { medical center. }\end{array}$ & 30 & $\begin{array}{c}\text { Mean } \\
57.97 \pm 18.05 \\
\text { Range } \\
20-80\end{array}$ & $\begin{array}{l}\text { On-road } \\
\text { testing }\end{array}$ \\
\hline & $\begin{array}{l}\text { Hargrave, } 2012 \\
\text { (U.S.) }^{(10)}\end{array}$ & $\begin{array}{l}\text { A study to examine } \\
\text { the utility of the } \\
\text { Frontal Assessment } \\
\text { Battery and the Trail } \\
\text { Making Test B in } \\
\text { predicting on-road } \\
\text { driving performance } \\
\text { after stroke or } \\
\text { traumatic brain injury. }\end{array}$ & $\begin{array}{l}\text { Participants were } \\
\text { referred for driving } \\
\text { assessment after } \\
\text { diagnosis of stroke or } \\
\text { traumatic brain injury } \\
\text { to a driving rehab } \\
\text { program. }\end{array}$ & 76 & $\begin{array}{c}\text { Mean } \\
57.3 \pm 17 \\
\text { Range } \\
18-87\end{array}$ & $\begin{array}{l}\text { On-road } \\
\text { testing }\end{array}$ \\
\hline & $\begin{array}{l}\text { Lundqvist, } 2008 \\
\text { (Sweden) }^{(60)}\end{array}$ & $\begin{array}{l}\text { A study to } \\
\text { examine long-term } \\
\text { consequences of } \\
\text { brain injury on } \\
\text { health status, driving } \\
\text { characteristics, and } \\
\text { car accidents and } \\
\text { to study whether } \\
\text { driving ten years } \\
\text { after brain injury was } \\
\text { retrospectively related } \\
\text { to cognitive function } \\
\text { and on-road driving } \\
\text { performance ten years } \\
\text { before. }\end{array}$ & $\begin{array}{l}\text { Cases were randomly } \\
\text { sampled from patients } \\
\text { treated for acquired } \\
\text { brain injury (from } \\
\text { TBI, subarachnoid } \\
\text { hemorrhage, stroke) at } \\
\text { a university hospital. } \\
\text { Source of healthy } \\
\text { matched controls not } \\
\text { clear. All held a valid } \\
\text { licence. }\end{array}$ & 80 & $\begin{array}{l}\text { Not reported for } \\
\qquad N=80\end{array}$ & Self-report \\
\hline
\end{tabular}


ROY:TRAILS B CUT-OFFS IN ASSESSING DRIVING FITNESS

TABLE 1.

Continued

\begin{tabular}{|c|c|c|c|c|c|c|}
\hline & $\begin{array}{c}\text { Author, Year } \\
\text { (Country) }\end{array}$ & $\begin{array}{l}\text { Description of } \\
\text { Study }\end{array}$ & $\begin{array}{c}\text { Description of } \\
\text { Participants }\end{array}$ & Sample Size & $\begin{array}{c}\text { Age in Years } \\
(\text { Mean } \pm S D \\
\text { Range })\end{array}$ & $\begin{array}{c}\text { Method of } \\
\text { Evaluating } \\
\text { Driving Safety }\end{array}$ \\
\hline \multirow[t]{2}{*}{$\begin{array}{l}\text { Traumatic } \\
\text { Brain Injury }\end{array}$} & $\begin{array}{l}\text { Novack, } 2006 \\
\text { (U.S.) }^{(61)}\end{array}$ & $\begin{array}{l}\text { A study to investigate } \\
\text { the relationship } \\
\text { between performance } \\
\text { on the Useful Field } \\
\text { of View test and } \\
\text { driving performance } \\
\text { following TBI. }\end{array}$ & $\begin{array}{l}\text { Participants were } \\
\text { referred for evaluation } \\
\text { by a physician to Dept. } \\
\text { of Rehab Services, } \\
\text { based on documented } \\
\text { progress following } \\
\text { TBI. All subjects had a } \\
\text { valid driver's license. } \\
\text { If participation in on- } \\
\text { road test was approved } \\
\text { by the driving } \\
\text { evaluator, client } \\
\text { consent was obtained. }\end{array}$ & 60 & $\begin{array}{c}\text { Mean } 33 \\
\text { Range } \\
16-68\end{array}$ & $\begin{array}{l}\text { On-road } \\
\text { testing }\end{array}$ \\
\hline & $\begin{array}{c}\text { Brooke, } 1992 \\
\left(_{(U . S .)}\right)^{(62)}\end{array}$ & $\begin{array}{l}\text { A study to examine } \\
\text { the relationship } \\
\text { between standardized } \\
\text { measures of cognitive } \\
\text { function and } \\
\text { measures of driving } \\
\text { performance in } \\
\text { patients with closed } \\
\text { head injuries and in } \\
\text { their age-matched } \\
\text { relative or friend } \\
\text { cohorts. }\end{array}$ & $\begin{array}{l}\text { Participants were } \\
\text { patients admitted to } \\
\text { a regional Level I } \\
\text { Trauma Center with } \\
\text { a diagnosis of closed } \\
\text { head injury 3-6 months } \\
\text { ago. Controls were } \\
\text { age-matched family } \\
\text { and friends of these } \\
\text { patients. }\end{array}$ & $\begin{array}{l}\mathrm{N}=20 \\
\text { Cases }=13(\mathrm{TBI}) \\
\text { Controls }=7(\mathrm{a} \\
\text { friend or relative } \\
\text { within } 5 \text { years of } \\
\text { the patient's age) }\end{array}$ & $\begin{array}{l}\text { Mean, SD, } \\
\text { and Range not } \\
\text { provided. The } \\
\text { only comment } \\
\text { on age of } \\
\text { participants is } \\
\text { range of age } \\
\text { in inclusion } \\
\text { criteria = } \\
18-65 .\end{array}$ & $\begin{array}{l}\text { On-road } \\
\text { testing }\end{array}$ \\
\hline Epilepsy & $\begin{array}{l}\text { Crizzle, } 2012 \\
\left(_{(U . S .)}\right)^{(63)}\end{array}$ & $\begin{array}{l}\text { A study to determine } \\
\text { which tests, from } \\
\text { a clinical battery, } \\
\text { are correlated with } \\
\text { driving errors in } \\
\text { people with epilepsy } \\
\text { using a simulator. }\end{array}$ & $\begin{array}{l}\text { Drivers with epilepsy } \\
\text { recruited from the } \\
\text { epilepsy monitoring } \\
\text { unit at a university } \\
\text { hospital. }\end{array}$ & 16 & $\begin{array}{c}\text { Mean } \\
44.3 \pm 12.0 \\
\text { Range } \\
22-68\end{array}$ & $\begin{array}{l}\text { Simulator } \\
\text { score }\end{array}$ \\
\hline
\end{tabular}

$\mathrm{AD}=$ Alzheimer Disease, $\mathrm{AD}-8=$ Aging and Dementia -8 questionnaire, $\mathrm{CDR}=$ Clinical Dementia Rating scale, $\mathrm{CNS}=\mathrm{Central}$ Nervous System, $\mathrm{DMV}=$ Department of Motor Vehicle, $\mathrm{HD}=$ Huntington Disease, MVA = Motor Vehicle Administration, MMSE $=$ Mini Mental State Examination, $\mathrm{MVC}=$ Motor Vehicle Collision, NINCDS-ADRDA = National Institute of Neurological and Communicative Disorders and Stroke and the Alzheimer's Disease and Related Disorders Association, PD = Parkinson's Disease, TBI = Traumatic Brain Injury. 


\section{ROY: TRAILS B CUT-OFFS IN ASSESSING DRIVING FITNESS}

TABLE 2.

Reported associations of Trails B with predicting driving safety (studies with no association shaded in gray)

\begin{tabular}{|c|c|c|c|}
\hline Author, Year (Country) & $\begin{array}{l}\text { Sample Size } \\
\text { (in ascending } \\
\text { order) }\end{array}$ & $\begin{array}{l}\text { Association of Trails B with } \\
\text { Predicting Driving Safety } \\
\text { (positive or no association) }\end{array}$ & Strength of Association \\
\hline Crizzle, 2012 (U.S.) $)^{(63)}$ & 16 & No association & - \\
\hline Fox, 1997 (Australia) ${ }^{(48)}$ & 19 & No association & - \\
\hline Brooke, 1992 (U.S.) ${ }^{(62)}$ & 20 & No association & - \\
\hline Szlyk, 2002 (U.S.) ${ }^{(36)}$ & 22 & Positive & $\begin{array}{l}\text { Correlation (Pearson or Spearman) } r=0.608, p=.004 \text { for } \\
\text { lane boundary crossing; } r=-0.571, p=.009 \text { for speed; } \\
r=-0.563, p=.01 \text { for brake pedal pressure }\end{array}$ \\
\hline Ott, 2003 (U.S.) ${ }^{(50)}$ & 27 & Positive & $\begin{array}{l}\mathrm{F}(1,22) \text { test }=6.03, p=.02 \text { for relation to caregiver } \\
\text { rating scale of driving ability }\end{array}$ \\
\hline Scally, 2011 Australia) $)^{(26)}$ & 28 & Positive & $\begin{array}{l}\text { Pearson correlation } r=0.61, p<.01 \text { for invalidly cued } \\
\text { braking point in Parkinson's Disease group and } r=0.59, \\
p<.01 \text { in control group; } r=0.58, p<.01 \text { for validly cued } \\
\text { braking point in control group }\end{array}$ \\
\hline Elkin-Frankston, 2007 (U.S.) ${ }^{(23)}$ & 29 & $\begin{array}{l}\text { No association } \\
\text { (with both Trails B and } \\
\text { Color Trails Test 2) }\end{array}$ & - \\
\hline Hartman-Maier, 2008 (Israel) ${ }^{(24)}$ & 30 & $\begin{array}{l}\text { No association }- \text { with } \\
\text { Color Trails Test } 2 . \\
\text { Does not look at Trails B. }\end{array}$ & - \\
\hline Rizzo, 2001 (U.S.) ${ }^{(49)}$ & 30 & Positive & $\begin{array}{l}\text { Odds Ratio } 13.47 \text { for crashes }(95 \% \text { CI } 1.19-747.68) \\
p=.016\end{array}$ \\
\hline Lundqvist, 2007 (Sweden) ${ }^{(57)}$ & 30 & No association & - \\
\hline Alexandersen, 2009 (Norway) $^{(56)}$ & 35 & No association & - \\
\hline Rizzo, 1997 (U.S.) ${ }^{(47)}$ & 39 & Positive & Odds Ratio 30.19 for crashes $\left(95 \%\right.$ CI $\left.3.8^{-\infty}\right), p<.001$ \\
\hline Whelihan, 2005 (U.S.) ${ }^{(55)}$ & 46 & Positive & $\begin{array}{l}\text { Zero-order correlation } r=0.46, p<.05 \text { for on-road } \\
\text { driving evaluation }\end{array}$ \\
\hline Soderstrom, 2006 (Sweden) $)^{(59)}$ & 54 & No association & - \\
\hline Petrakos, 2009 (U.S.) $^{(31)}$ & 57 & No association & - \\
\hline Novack, 2006 (U.S.) ${ }^{(61)}$ & 60 & Positive & $\begin{array}{l}\text { Standardized regression coefficient }=0.29(p<.05) \text { for } \\
\text { predictor of global driving evaluation rating and } 0.03 \text { for } \\
\text { observer-rated Driving Assessment Scale score }\end{array}$ \\
\hline Devos, 2012 (Belgium) ${ }^{(58)}$ & 60 & Positive & Wilcoxon rank sum test $\mathrm{W}=301, p=.009$ \\
\hline Grace, 2005 (U.S.) $)^{(46)}$ & 62 & Positive & $\mathrm{F}(1.34)=13.05, p=.001$ for on-road driving test \\
\hline Hargrave, 2012 (U.S.) ${ }^{(10)}$ & 76 & Positive & $\begin{array}{l}\text { Odds Ratio } 1.012, p<.05 \text { for on-road driving evalua- } \\
\text { tion outcome }\end{array}$ \\
\hline Niewoehner, 2012 (U.S.) $^{(30)}$ & 77 & Positive & $\begin{array}{l}p<.001 \text { for on-road driving test; Pearson correlation } \\
\text { coefficients done but not reported. }\end{array}$ \\
\hline Lundqvist, 2008 (Sweden) ${ }^{(60)}$ & 80 & No association & - \\
\hline
\end{tabular}


TABLE 2.

Continued

\begin{tabular}{|c|c|c|c|}
\hline Author, Year (Country) & $\begin{array}{l}\text { Sample Size } \\
\text { (in ascending } \\
\text { order) }\end{array}$ & $\begin{array}{l}\text { Association of Trails B with } \\
\text { Predicting Driving Safety } \\
\text { (positive or no association) }\end{array}$ & Strength of Association \\
\hline Mazer, 1998 (Canada) $^{(14)}$ & 84 & Positive & $\begin{array}{l}\text { Odds Ratio } 5.96 \text { (CI 1.83-19.42), } p<.01 \text { for on-road } \\
\text { driving evaluation; Positive Predictive Value }=85.2 \% \text {, } \\
\text { Negative Predictive Value }=48.1 \%\end{array}$ \\
\hline Carr, 2011 (U.S.) $^{(54)}$ & 85 & Positive & $p<.001$ for on-road driving evaluation outcome \\
\hline Selander, 2011 (Sweden) $)^{(44)}$ & 85 & No association & - \\
\hline Emerson, 2012 (U.S.) ${ }^{(40)}$ & 100 & Positive & $\begin{array}{l}\text { Hazard Ratio } 1.40 \text { ( } 95 \% \text { CI } 1.06-1.84), p<.05 \text { for ability } \\
\text { to predict future crashes. }\end{array}$ \\
\hline Park, 2011 (Korea) $^{(43)}$ & 103 & Positive & None provided \\
\hline Bliokas, 2011 (Australia) $^{(17)}$ & 104 & Positive & $\begin{array}{l}\text { Pearson's } r=0.28(p<.01) \text { for number of corrective } \\
\text { interventions performed by driving instructor during } \\
\text { on-road test; Spearman rho }=0.32(p<.01) \text { for pass/ } \\
\text { fail on road test }\end{array}$ \\
\hline Tarawneh, 1993 (U.S.) ${ }^{(39)}$ & 105 & Positive & $\begin{array}{l}\text { Correlation coefficient }-0.42, p=.0001 \text { for on-road } \\
\text { driving performance }\end{array}$ \\
\hline Freund, 2008 (U.S.) $)^{(32)}$ & 108 & Positive & $\begin{array}{l}\mathrm{F}(2,76)=9.96, p<.001 \text { for driving simulator perfor- } \\
\text { mance }\end{array}$ \\
\hline Ott, 2008 (U.S.) $^{(53)}$ & 121 & Positive & $\begin{array}{l}\text { Pearson's } r=0.48, p<.0005 \text { for on-road driving evalu- } \\
\text { ation score }\end{array}$ \\
\hline Cushman, 1996 (U.S.) $)^{(38)}$ & 123 & Positive & $t=7.10, p<.001$ for on-road driving performance \\
\hline Marottoli, 1998 (U.S.) $)^{(11)}$ & 125 & Positive & Hazard Ratio 1.42 for self-reported events \\
\hline Classen, 2008 (U.S.) ${ }^{(16)}$ & 127 & Positive & $\begin{array}{l}\text { Odds Ratio } 2.5 \text { (95\% CI 1.0-5.9) for failing on-road } \\
\text { driving test }\end{array}$ \\
\hline Betz, 2009 (U.S.) ${ }^{(15)}$ & 144 & No association & - \\
\hline Dawson, 2009 (U.S.) ${ }^{(45)}$ & 155 & No association & - \\
\hline O’Connor, 2010 (U.S.) $)^{(42)}$ & 160 & Positive & $p<.001$ for on-road driving evaluation outcome \\
\hline Uc, 2005 (U.S.) $)^{(51)}$ & 170 & Positive & $\begin{array}{l}\text { Spearman correlation } r=-0.45, p<.0001 \text { for Landmark } \\
\text { and Traffic Identification Test on a driving simulator }\end{array}$ \\
\hline Freund, 2008 (U.S.) ${ }^{(33)}$ & 176 & No association & - \\
\hline Uc, 2006 (U.S.) $)^{(52)}$ & 176 & Positive & $\begin{array}{l}\text { Odds Ratios for unsafe outcomes on driving simulator: } \\
1.22(95 \% \text { CI } 1.01-1.46) \text { for crash or risky avoidance } \\
\text { behaviour, } 1.31 \text { (95\% CI } 1.12-1.54) \text { for abrupt slowing, } \\
1.17 \text { (95\% CI } 1.02-1.35) \text { for premature stopping }\end{array}$ \\
\hline Uc, 2006 (U.S.) $)^{(25)}$ & 230 & Positive & $\begin{array}{l}\text { Spearman correlation } r=0.35, p<.01 \text { for Trails B-A for } \\
\text { at-fault safety errors on driving simulator }\end{array}$ \\
\hline Wood, 2008 (Australia) $^{(34)}$ & 270 & Positive & $\begin{array}{l}t(55.6)=-3.15, p<.01 \text { for on-road driving evaluation } \\
\text { outcome }\end{array}$ \\
\hline Rozzini, 2012 (Italy) $)^{(41)}$ & 297 & Positive & $\begin{array}{l}\text { Odds Ratio } 2.3(95 \% \text { CI } 1.06-4.9), p<.03 \text { for self- } \\
\text { reported crash }\end{array}$ \\
\hline
\end{tabular}


TABLE 2.

Continued

\begin{tabular}{|c|c|c|c|}
\hline Author, Year (Country) & $\begin{array}{l}\text { Sample Size } \\
\text { (in ascending } \\
\text { order) }\end{array}$ & $\begin{array}{l}\text { Association of Trails } B \text { with } \\
\text { Predicting Driving Safety } \\
\text { (positive or no association) }\end{array}$ & Strength of Association \\
\hline Kantor, 2004 (U.S.) ${ }^{(29)}$ & 664 & $\begin{array}{l}\text { Positive } \\
\text { Reports positive association } \\
\text { as cues needed to complete Trails } \\
\text { B - methodology for determining } \\
\text { "cue score" was not mentioned. }\end{array}$ & $\begin{array}{l}\text { Statistical analysis for Trails B alone not provided in } \\
\text { clear terms }\end{array}$ \\
\hline $\begin{array}{l}\text { Staplin, } 2003 \text { (U.S.) } \\
\quad \text { (original MaryPODS data) }^{(13)}\end{array}$ & 1876 & $\begin{array}{l}\text { Positive } \\
\text { The original data included two } \\
\text { years of prospective crash data. }\end{array}$ & $\begin{array}{l}\text { Odds Ratio } 3.50, p<.01 \text { for at-fault crashes; Odds Ratio } \\
1.72, p<.01 \text { for frequencies of violations }\end{array}$ \\
\hline $\begin{array}{l}\text { Staplin, } 2003 \text { (U.S.) } \\
\quad \text { (updated MaryPODS data) }^{(35)}\end{array}$ & 1876 & $\begin{array}{l}\text { No association } \\
\text { This updated analysis included } \\
\text { one additional year of driving } \\
\text { experience. }\end{array}$ & - \\
\hline Ball, 2006 (U.S.) ${ }^{(12)}$ & 1910 & Positive & $\begin{array}{l}\text { Odds Ratio } 1.21 \text { ( } 95 \% \text { CI 1.01-1.44), } p=.04 \text { for future } \\
\text { at-fault crashes }\end{array}$ \\
\hline Stutts, 1998 (U.S.) $)^{(37)}$ & 3238 & Positive & $\begin{array}{l}\text { Odds Ratio } 1.06 \text { (95\% CI 1.01-1.11) for crash involve- } \\
\text { ment }\end{array}$ \\
\hline
\end{tabular}

TABLE 3.

Studies reporting Trails B cut-off values

\begin{tabular}{|c|c|c|}
\hline Author, Year (Country) & Reported Trails B Cut-off Value & Source of Reported Cut-off \\
\hline Hargrave, 2012 (U.S.) ${ }^{(10)}$ & 90 seconds & Analysis of primary driving research \\
\hline Marottoli, 1998 (U.S.) ${ }^{(11)}$ & 133 seconds & \\
\hline Ball, 2006 (U.S.) ${ }^{(12)}$ & 147 seconds & \\
\hline Staplin, 2003 (U.S.) (original MaryPODS data) $)^{(13)}$ & 180 seconds & \\
\hline Mazer, 1998 (Canada) $^{(14)}$ & $<3$ errors & \\
\hline Betz, 2009 (U.S.) $^{(15)}$ & 180 seconds & References (Wang $2003^{(18)}$ and Tombaugh $\left.2004^{(6)}\right)^{\mathrm{a}}$ \\
\hline Classen, 2008 (U.S.) ${ }^{(16)}$ & 3 minutes & $\begin{array}{l}\text { References (Fals-Stewart 1992(20) } \\
\left.\text { and Franzen } 1996^{(21)}\right)^{\mathrm{a}}\end{array}$ \\
\hline Bliokas, 2011 (Australia) ${ }^{(17)}$ & $\geq 292$ seconds & Reference (Lezak $\left.1983^{(19)}\right)^{\mathrm{a}}$ \\
\hline
\end{tabular}

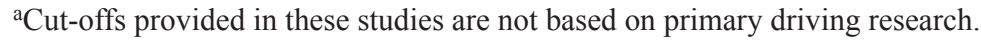

It is also critical that tests such as Trails B not be misused - they must be accurately interpreted in the context of a number of critical considerations, in order to ensure that they are a valid reflection of function. ${ }^{(1)}$ In order to avoid generating false results, Trails B scores should always be interpreted in the overall clinical context when determining fitness-to-drive. ${ }^{(7)}$ The clinician should confirm that the Trails B results are consistent with the history provided by caregivers and other tests. Low scores must be verified as not to be due to confounding variables such as language barrier, low education, dyslexia, performance anxiety, depression, or sensory deficits, for example.

The administration of Trails B should also be standardized, as cognitive performance can be influenced by many factors. Ideally, all assessors should receive identical instructions on test administration. A practical recommendation may be that assessors receive training through continuing medical education. 
For a review of considerations in applying in-office tests to the assessment of fitness-to-drive, please see page 11 of $\mathrm{http} / /$ www.canadiangeriatrics.ca/default/index.cfm/ linkservid/0D194943-EF73-7DAB-77450BB92BFF239A/ showMeta/0/. ${ }^{(7)}$ Furthermore, tests such as Trails B can be employed within a more detailed assessment process, as described in http://www.cfp.ca/content/56/11/1123.full. pdf + html?sid=6ddf379a-874a-4d6f-9c64-02c6bf939312. ${ }^{(2)}$

The evidence from the Tombaugh article $^{(6)}$ (that the mean Trails B score for all age groups is $<3$ minutes and only a small number of outliers have Trails $\mathrm{B}>3$ minutes) and the articles listing fitness-to-drive cut-offs of 3 minutes or 3 errors, ${ }^{(15,13,16,14)}$ support the finding that the best evidence-informed cut-offs we have to date are 3 minutes or 3 errors, as described in three continuing medical education articles. $^{(2,7,8)}$

In this systematic review, none of the studies justified sample sizes via formal calculations. Eleven of the 15 studies which showed no association between Trails B and driving had small sample sizes of $\leq 100$. Due to the risk of type II (beta) errors (i.e., false negative results caused by inadequate sample size or insufficient power), the findings of these 11 small studies cannot be interpreted with any degree of confidence (i.e., we cannot tell if they are true negative or false negative studies). This concern may also be true for the additional three negative studies with sample sizes ranging from 144 to 176 .

A limitation of the Trail Making Test is that it requires knowledge of the numbers and letters used in the English language and, thus, may not be appropriate for individuals whose primary language does not employ similar letters and numbers or those who are illiterate. One instrument that has been developed to address this concern is the Color Trails Test (CTT). The CTT is a language-free analogue of the Trails test designed to be applicable across various cultural contexts. Two studies ${ }^{(23,24)}$ (Table 2) looked at the CTT and its association with ability to predict fitness-to-drive. CTT 2 is similar to Trails B. It has two sets of 25 numbers in yellow and pink circles with instructions to connect the numbers in ascending order alternating between the two color sets. Both studies failed to show an association between CTT 2 and driving. However, it should once again be noted that both studies had small sample sizes $(\mathrm{N}=29$ and 30$)$ and did not show sample size calculations. Therefore, as discussed above, this could have created possible false negative results in both studies.

\section{CONCLUSION}

While the evidence for Trails B cut-offs of 3 minutes or 3 errors (the ' 3 or 3 rule') is limited, this systematic review reveals that these represent the best evidence-informed cut-offs available to date. It is logical to assume that as the test score worsens (e.g., the time to completion and/or the numbers of errors increase), the person's fitness-to-drive also worsens (i.e., risk of crash increases). It is, at the very least, reasonable for physicians to consider reporting findings to their Ministry of Transportation if the Trails B score is worse than 3 minutes or 3 errors, provided the test results are felt to be a valid reflection of function.

The body of evidence for Trails B cut-off scores is limited, in part, due to major methodological limitations of driving research uncovered in this study including: (1) lack of justification of sample size making the interpretation of small negative trials impossible as some negative findings may represent Type II or Beta Error (i.e., falsely negative findings due to inadequate sample size/insufficient power); and (2) the fact that most research is focused on associations but often ignores the derivation of cut-off scores, resulting in findings that are not clinically useful.

Not only is more research into Trails B cut-offs needed, but the quality of the research being done (i.e., the methodological standards) must improve. Recommendations for future driving research should therefore include:

1. The determination of sample size to prevent future small studies from reporting potentially falsely negative findings due to inadequate sample size/insufficient power (Type II or Beta Error). The fact that such sample size calculations are challenging does not justify their exclusion.

2. The determination of potential clinically useful cut-off scores using Receiver Operating Characteristic (ROC) curve analytic techniques that plot sensitivity vs. 1 specificity to permit the evaluation of the properties (e.g., sensitivity and specificity) of all potential cut-offs.

3. Given that there are likely no perfect cut-off scores with perfect sensitivity and specificity, techniques (e.g., Delphi techniques) that balance the risks and benefits of different cut-off scores, derived from ROC analyses, should be incorporated into driving research. Ultimately decisions regarding the best cutoffs need to be based on balancing the risks of missing cases of unsafe drivers vs. the risk of inappropriate loss of driving privileges.

4. Exploring the use of two cut-off scores to promote Trichotomization - see page 11 of http://www.canadian geriatrics.ca/default/index.cfm/linkservid/0D194943EF73-7DAB-77450BB92BFF239A/showMeta/0/(7) and http://onlinelibrary.wiley.com/doi/10.1111/j.15325415.2006.00967.x/pdf. ${ }^{(22)}$

5. Exploring different scoring methods for Trails $B$ such as Trails (B-A) ${ }^{(25,26,27,28)}$ and Trails B/A. ${ }^{(29)}$ Trails (B-A) has been described as reflecting "the attention and setswitching components of Trails B independent of psychomotor speed"(26) and is often considered the standard index of set-shifting. It is also "a measure of global executive function".(27) It has been examined in various driving studies with Parkinson's Disease patients, ${ }^{(25,26,27)}$ and has been found to be a good predictor of driving 
safety. It is thought that "the flexibility of the cognitive system", as tested by Trails B-A, "allows drivers to cope with dynamic traffic situations". ${ }^{(28)}$ Although it is certainly a measure that is worth examining, we chose not to investigate cut-off scores for Trails (B-A) in this systematic review because current guidelines from medical associations recommend the use of Trails B only, not Trails B-A.

6. Different forms of Trails B that can overcome literacy barriers such as Color Trails. ${ }^{(23,24)}$

In fact, we do not need to wait to add to this body of evidence. Researchers who have previously published Trails $\mathrm{B}$ research (or their MSc and $\mathrm{PhD}$ students) can immediately study the following in their existing databases: i) dichotomization via single cut-off scores (both time and number of errors), ii) trichotomization via two cut-off scores (both time and number of errors), and iii) novel scoring methods such as Trails $(\mathrm{B}-\mathrm{A})$ and Trails B/A.

\section{ACKNOWLEDGEMENTS}

We wish to thank Debbie Ayotte, librarian, for her extensive assistance with literature searches.

\section{CONFLICT OF INTEREST DISCLOSURES}

The authors declare that no conflicts of interest exist. Neither author has received financial support from private industry for work on the assessment of fitness-to-drive. Both authors were involved in the preparation of this manuscript.

\section{REFERENCES}

1. CMA. CMA driver's guide: determining medical fitness to operate motor vehicles (8th Edition). Available from: http:// www.cma.ca/driversguide

2. Molnar F, Simpson C. Approach to assessing fitness to drive in patients with cardiac and cognitive conditions. Can Fam Physician. 2010;56(11):1123-29. Available from: http://www. cfp.ca/content $/ 56 / 11 / 1123$.full.pdf + html? sid=a5434c3c-fa40 4c13-b5fc-3297d24d60e6

3. Canadian Council of Motor Transport Administrators. Determining driver fitness in Canada: Part 1: A model for the administration of driver fitness programs and Part 2: CCMTA medical standards for drivers (Version: Draft 8, 2011). Available from: http://www.ccmta.ca/english/pdf/medical_admin process_part_1_may2011.pdf

4. Driver and Vehicle Licensing Agency. Review of medical driver licensing (February 2006). Available from: http://www. dft.gov.uk/dvla/medical/medical_advisory information/ reviewofdm.aspx

5. American Medical Association. Physician's guide to assessing and counseling older drivers (September 2003). Available from: http://www.nhtsa.dot.gov/people/injury/olddrive/ OlderDriversBook
6. Tombaugh T. Trail Making Test A and B: normative data stratified by age and education. Arch Clin Neuropsychol. 2004;19(2):203-14.

7. Molnar F, Rapoport M, Roy M. Dementia and driving: maximizing the utility of in-office screening and assessment tools. CGS. 2012;2(2):11-14. Available from: http://www.canadian geriatrics.ca/default/index.cfm/linkservid/0D194943-EF737DAB-77450BB92BFF239A/showMeta/0/

8. Byszewski A, Molnar F, Rapoport M, et al. Practical experience-based approaches to assessing fitness to drive in dementia. Geriatr Aging. 2009;12(2):83-92.

9. Moher D, Liberati A, Tetzlaff J, et al. Preferred reporting items for systematic reviews and meta-analyses: the PRISMA statement. Ann Intern Med. 2009;151(4):264-69.

10. Hargrave D, Nupp J, Erickson R. Two brief measures of executive function in the prediction of driving ability after acquired brain injury. Neuropsychol Rehabil. 2012;22(4):489-500.

11. Marottoli R, Richardson E, Stowe M, et al. Development of a test battery to identify older drivers at risk for self-reported adverse driving events. J Am Geriatr Soc. 1998;46(5):562-68.

12. Ball KK, Roenker DL, Wadley VG, et al. Can high-risk older drivers be identified through performance-based measures in a Department of Motor Vehicles setting? J Am Geriatr Soc. 2006;54(1):77-84.

13. Staplin L, Lococo K, Gish K, et al. Model driver screening and education program. Final technical report. Volume 2: Maryland Pilot Older Driver Study. Report no. DOT HS 809 583. Washington (DC): National Highway Traffic Safety Administration; 2003.

14. Mazer B, Korner-Bitensky N, Sofer S. Predicting ability to drive after stroke. Arch Phys Med Rehabil. 1998;79(7):743-50.

15. Betz M, Fisher J. The Trail-Making Test B and driver screening in the emergency department. Traffic Inj Prev. 2009; 10(5):415-20.

16. Classen S, Horgas A, Awadzi K, et al. Clinical predictors of older driver performance on a standardized road test. Traffic Inj Prev. 2008;9(5):456-62.

17. Bliokas V, Taylor J, Leung J, et al. Neuropsychological assessment of fitness to drive following acquired cognitive impairment. Brain Injury 2011; 25(5): 471-487.

18. Wang C, Kosinski C, Schwartzberg J, et al. AMA physician's guide to assessing and counseling older drivers. Washington (DC): National Highway Traffic Safety Administration and American Medical Association; 2003. Available from: http:// www.ama-assn.org/ama/pub/physician-resources/publichealth/promoting-healthy-lifestyles/geriatric-health/olderdriver-safety/assessing-counseling-older-drivers.page

19. Lezak M. Neuropsychological assessment, 2nd ed. New York: Oxford University Press; 1983.

20. Fals-Stewart W. An interrater reliability study of the Trail Making Test (parts A and B). Perceptual Motor Skills. 1992;74(1):39-42.

21. Franzen M, Paul D, Iverson G. Reliability of alternate forms of the trail making test. Clin Neuropsychol. 1996;10(2):125-29. 
22. Molnar F, Patel A, Marshall S, et al. Clinical utility of officebased predictors of fitness to drive in persons with dementia: a systematic review. J Am Geriatr Soc. 2006;54(12):1809-24. Available from: http://onlinelibrary.wiley.com/doi/10.1111/ j.1532-5415.2006.00967.x/pdf

23. Elkin-Frankston S, Lebowitz B, Kapust L, et al. The use of the Color Trails Test in the assessment of driver competence: preliminary report of a culture-fair instrument. Arch Clin Neuropsychol. 2007;22(5):631-35.

24. Hartman-Maeir A, Bar-Haim Erez A, Ratzon N, et al. The validity of the Color Trail Test in the pre-driver assessment of individuals with acquired brain injury. Brain Inj. 2008;22(1314):994-98.

25. Uc E, Rizzo M, Anderson S, et al. Impaired visual search in drivers with Parkinson's disease. Ann Neurol. 2006;60(4):407-13.

26. Scally $\mathrm{K}$, Charlton J, Iansek R, et al. Impact of external cue validity on driving performance in Parkinson's disease. Parkinson's Dis. 2011. Epub 2011 Jun 8.

27. Ranchet M, Paire-Ficout L, Marin-Lamellet C, et al. Impaired updating ability in drivers with Parkinson's disease. J Neurol Neurosurg Psychiatry. 2011;82(2):218-23.

28. Adrian J, Postal V, Moessinger M, et al. Personality traits and executive functions related to on-road driving performance among older drivers. Accid Anal Prev. 2011;43(5):1652-59.

29. Kantor, B, Mauger L, Richardson V, et al. An analysis of an older driver evaluation program. J Am Geriatr Soc. 2004;52(8):1326-30.

30. Niewoehner P, Henderson R, Dalchow J, et al. Predicting road test performance in adults with cognitive or visual impairment referred to a veterans affairs medical center driving clinic. $J$ Am Geriatr Soc. 2012;60(11):2070-74.

31. Petrakos D, Freund B. Driving habits of older drivers 3 months before driving evaluation. Topic Geriatr Rehabil. 2009;25(2):118-34.

32. Freund B, Colgrove L. Error specific restrictions for older drivers: promoting continued independence and public safety. Accid Anal Prev. 2008;40(1):97-103.

33. Freund $\mathrm{B}$, Colgrove $\mathrm{L}$, Petrakos $\mathrm{D}$, et al. In my car the brake is on the right: pedal errors among older drivers. Accid Anal Prev. 2008;40(1):403-09.

34. Wood J, Anstey K, Kerr G, et al. A multidomain approach for predicting older driver safety under in-traffic road conditions. $J$ Am Geriatr Soc. 2008;56(6):986-93.

35. Staplin L, Gish K, Wagner E. MaryPODS revisited: updated crash analysis and implications for screening program implementation. J Safety Res. 2003;34(4):389-97.

36. Szlyk J, Myers L, Zhang Y, et al. Development and assessment of a neuropsychological battery to aid in predicting driving performance. J Rehabil Res Dev. 2002;39(4):483-96.

37. Stutts J, Stewart J, Martell C. Cognitive test performance and crash risk in an older driver population. Accid Anal Prev. 1998;30(3):337-46.

38. Cushman L. Cognitive capacity and concurrent driving performance in older drivers. IATSS Res. 1996;20(1):38-45.
39. Tarawneh M, McCoy P, Bishu R, et al. Factors associated with driving performance of older drivers. Transportation Res Record. 1993;1405:64-71.

40. Emerson J, Johnson A, Dawson J, et al. Predictors of driving outcomes in advancing age. Psychol Aging. 2012;27(3):550-59.

41. Rozzini L, Riva M, Zanetti M, et al. The impact of cognitive deficit on self-reported car crashes in ultra-octogenarian population: data of an Italian population-based study. Int J Geriatr Psychiatry. 2012;28(6):562-66.

42. O'Connor M, Kapust L, Lin B, et al. The 4Cs (crash history, family concerns, clinical condition, and cognitive functions): a screening tool for the evaluation of the at-risk driver. $J \mathrm{Am}$ Geriatr Soc. 2010;58(6):1104-08.

43. Park S, Choi E, Lim M, et al. Association between unsafe driving performance and cognitive-perceptual dysfunction in older drivers. PM R. 2011;3(3):198-203.

44. Selander H, Lee H, Johansson K, et al. Older drivers: on-road and off-road test results. Accid Anal Prev. 2011;43(4):1348-54.

45. Dawson J, Anderson S, Uc E, et al. Predictors of driving safety in early Alzheimer disease. Neurology. 2009;72(6):521-27.

46. Grace J, Amick M, D’Abreau A, et al. Neuropsychological deficits associated with driving performance in Parkinson's and Alzheimer's disease. J Int Neuropsychol Soc. 2005;11(6):766-75.

47. Rizzo M, Reinach S, McGehee D, et al. Simulated car crashes and crash predictors in drivers with Alzheimer disease. Arch Neurol. 1997;54(5):545-51.

48. Fox G, Bowden S, Bashford G, et al. Alzheimer's disease and driving: prediction and assessment of driving performance. $J$ Am Geriatr Soc. 1997;45(8):949-53.

49. Rizzo M, McGhee D, Dawson J, et al. Simulated car crashes at intersections in drivers with Alzheimer disease. Alzheimer Dis Assoc Disord. 2001;15(1):10-20.

50. Ott B, Heindel W, Whelihan W, et al. Maze test performance and reported driving ability in early dementia. J Geriatr Psychiatry Neurol. 2003;16(3):151-55.

51. Uc E, Rizzo M, Anderson S, et al. Driver landmark and traffic sign identification in early Alzheimer's disease. J Neurol Neurosurg Psychiatry. 2005;76(6):764-68.

52. Uc E, Rizzo M, Anderson S, et al. Unsafe rear-end collision avoidance in Alzheimer's disease. J Neurol Sci. 2006;251(12):35-43.

53. Ott B, Festa E, Amick M, et al. Computerized maze navigation and on-road performance by drivers with dementia. J Geriatr Psychiatry Neurol. 2008;21(1):18-25.

54. Carr D, Barco P, Wallendorf $\mathrm{M}$, et al. Predicting road test performance in drivers with dementia. J Am Geriatr Soc. 2011;59(11):2112-17.

55. Whelihan W, DiCarlo M, Paul R. The relationship of neuropsychological functioning to driving competence in older persons with early cognitive decline. Arch Clin Neuropsychol. 2005;20(2):217-28.

56. Alexandersen A, Dalen K, Bronnick K. Prediction of driving ability after inconclusive neuropsychological investigation. Brain Inj. 2009;23(4):313-21. 
57. Lundqvist A, Alinder J. Driving after brain injury: selfawareness and coping at the tactical level of control. Brain Inj. 2007;21(11):1109-17.

58. Devos H, Nieuwboer A, Tant M, et al. Determinants of fitness to drive in Huntington disease. Neurology. 2012;79(19):1975-82.

59. Soderstrom S, Pettersson R, Leppert J. Prediction of driving ability after stroke and the effect of behind-the-wheel training. Scand J Psychol. 2006;47(5):419-29.

60. Lundqvist A, Alinder J, Ronnberg J. Factors influencing driving 10 years after brain injury. Brain Inj. 2008;22(4):295-304.

61. Novack T, Banos J, Alderson A, et al. UFOV performance and driving ability following traumatic brain injury. Brain Inj. 2006;20(5):455-61.
62. Brooke M, Questad K, Patterson D, et al. Driving evaluation after traumatic brain injury. Am J Phys Med Rehabil. 1992;71(3):177-82.

63. Crizzle A, Classen S, Winter S, et al. Associations between clinical tests and simulated driving performance in persons with epilepsy. Epilepsy Behav. 2012;23(3):241-46.

Correspondence to: Dr. Frank J. Molnar, MD, FRCPC, University of Ottawa Division of Geriatric Medicine, The Ottawa Hospital, Civic Campus, 1053 Carling Ave, Ottawa, ON K1Y 4E9, Canada

E-mail: fmolnar@ottawahospital.on.ca 